\title{
THE UNIQUENESS OF SOLUTIONS OF THE HEAT EQUATION IN AN INFINITE STRIP
}

\author{
BY \\ VICTOR L. SHAPIRO( ${ }^{(1)}$
}

1. Introduction. We shall say $u(x, t)$ is a solution of the heat equation in the strip $0<t<c$ if $u(x, t)$ is in class $C^{2}$ in this strip and if $u_{x x}(x, t)=u_{t}(x, t)$ for every point $(x, t)$ of this strip. We shall designate the fundamental solution of the heat equation for $t>0$ by $k(x, t)=(4 \pi t)^{-1 / 2} \exp \left[-x^{2} / 4 t\right]$ and shall prove in this paper the following theorem:

THEOREM 1. Let $u(x, t)$ be a solution of the heat equation in the strip $0<t<c$. Suppose that

(i) $|u(x, t)| \leqq \varepsilon(t) \exp \left[\alpha x^{2}\right] t^{-1}$ for $0<t<c$ where $\alpha$ is a positive constant and $\varepsilon(t)$ is bounded for $0<t<c$ and $\varepsilon(t)=o(1)$ as $t \rightarrow 0$;

(ii) $\lim \sup _{t \rightarrow 0}|u(x, t)|$ is finite-valued except possibly for a countable set $E$;

(iii) $\lim \inf _{t \rightarrow 0} u(x, t)=u_{*}(x)$ is in $L^{1}$ on every compact subset of $(-\infty, \infty)$;

(iv) there exist positive constants $K$ and $\beta$ such that $|U(x)| \leqq K \exp \left[\beta x^{2}\right]$ where $U(x)=\int_{0}^{x} \int_{0}^{y} u_{*}(z) d z d y$;

(v) $\lim \inf _{t \rightarrow 0} t^{1 / 2} u(x, t)=0$ for $x$ in $E$.

Then

$$
u(x, t)=\int_{-\infty}^{\infty} k_{t}(x-y, t) U(y) d y \quad \text { for } \quad 0<t<\min \left[c,(4 \beta)^{-1}\right]
$$

As a corollary to Theorem 1, we get the following uniqueness theorem for solutions of the heat equation stated in a more conventional form:

THEOREM 2. Let $u(x, t)$ be a solution of the heat equation in the strip $0<t<c$. Suppose that

(i) $|u(x, t)| \leqq \varepsilon(t) \exp \left[\alpha x^{2}\right] t^{-1}$ for $0<t<c$ where $\alpha$ is a positive constant and $\varepsilon(t)$ is bounded for $0<t<c$ and $\varepsilon(t)=o(1)$ as $t \rightarrow 0$;

(ii) $\lim _{\sup _{t \rightarrow 0}}|u(x, t)|$ is finite-valued except possibly for a countable set $E$;

(iii) $\lim \inf _{t \rightarrow 0} u(x, t)=u_{*}(x)$ is in $L^{1}$ on $(-\infty, \infty)$;

(iv) $\lim \inf _{t \rightarrow 0} t^{1 / 2} u(x, t)=0$ for $x$ in $E$.

Then $u(x, t)=\int_{-\infty}^{\infty} k(x-y, t) u_{*}(y) d y$ for $0<t<c$.

Presented to the Society, April 10, 1965; received by the editors September 8, 1965 and, in revised form, May 13, 1966.

( ${ }^{1}$ This research was supported by the Air Force Office of Scientific Research. 
To see that Theorem 2 is indeed a corollary to Theorem 1 , choose $t_{0}$ such that $0<t_{0}<c$. Then we need only show the conclusion of Theorem 2 holds in the strip $0<t<t_{0}$. Now if $u_{*}(x)$ is in $L^{1}$ on $(-\infty, \infty)$, then $U(x)$ defined in (iv) of Theorem 1 is such that $|U(x)| \leqq K_{1}|x| \leqq K_{2} \exp \left[x^{2} / 4 t_{0}\right]$ where $K_{1}$ and $K_{2}$ are constants. Consequently, given (i), (ii), (iii), and (iv) of Theorem 2, we have by Theorem 3 that

$$
u(x, t)=\int_{-\infty}^{\infty} k_{t}(x-y, t) U(y) d y \quad \text { for } 0<t<t_{0} .
$$

Now, fixing $x$ in this equality, observing that $\partial k(x-y, t) / \partial t=\partial^{2} k(x-y, t) / \partial y^{2}$ for $t>0$, and integrating by parts twice in the above integral, we obtain that

$$
u(x, t)=\int_{-\infty}^{\infty} k(x-y, t) u_{*}(y) d y \quad \text { for } 0<t<t_{0}
$$

which establishes Theorem 2 .

Now both Theorems 1 and 2 were motivated by Theorem 3 to be stated below. The interesting fact concerning Theorem 3 is that each of the three conditions comprising its hypothesis can be shown to be in a certain sense best possible.

For $t>0$, we define $\|u(x, t)\|_{\infty}=\sup _{-\infty<x<\infty}|u(x, t)|$ and state Theorem 3 as follows:

THEOREM 3. Let $u(x, t)$ be a solution of the heat equation in the strip $0<t<c$ and be bounded in every substrip of the form $0<t_{0} \leqq t<c$. Suppose that

(i) $\|u(x, t)\|_{\infty}=o\left(t^{-1}\right)$ as $t \rightarrow 0$;

(ii) $\lim _{t \rightarrow 0} u(x, t)=0$ except possibly for a countable set $E$;

(iii) $\lim \inf _{t \rightarrow 0} t^{1 / 2} u(x, t)=0$ for every $x$ in $E$.

Then $u(x, t)$ is identically zero in the strip $0<t<c$.

We see that Theorem 3 is an immediate corollary of Theorem 2. To show that each of three conditions in the hypothesis of Theorem 3 is in a certain sense best possible we first note that both

$$
k(x, t)=(4 \pi t)^{-1 / 2} \exp \left[-x^{2} / 4 t\right]
$$

and

$$
k_{x}(x, t)=-\left(4 \pi^{1 / 2} t^{3 / 2}\right)^{-1} x \exp \left[-x^{2} / 4 t\right]
$$

are solutions of the heat equation in every strip of the form $0<t<c$ and both are bounded in any substrip of the form $0<t_{0} \leqq t<c$.

To show that the conclusion of Theorem 3 does not hold if (i) is weakened by replacing " $o$ " by " $O$," we observe that $\left\|k_{x}(x, t)\right\|_{\infty}=O\left(t^{-1}\right)$ as $t \rightarrow 0$. Furthermore $k_{x}(x, t)$ meets (ii) and (iii) since $\lim _{t \rightarrow 0} k_{x}(x, t)=0$ for every $x$. We conclude that condition (i) as stated is in a certain sense best possible. 
To show that (ii) of Theorem 3 cannot be weakened by replacing "except possibly for a countable set $E$ " by "except possibly for an uncountable Borel set $E$ of measure zero," we observe that if $E$ is an uncountable Borel set, then there exists a bounded perfect set $Z \subset E$ [1, p. 205] of measure zero. Consequently, there exists a continuous (i.e., nonatomic) probability measure $\mu$ with its support contained in $Z$ [2, p. 101].

We set

$$
u(x, t)=\int_{-\infty}^{\infty} k(x-y, t) d \mu(y) \quad \text { for } t>0,
$$

and observe that $u(x, t)$ is a solution of the heat equation for $t>0$ and is bounded in every substrip of the form $0<t_{0} \leqq t<\infty$. Also we see that $\lim _{t \rightarrow 0} u(x, t)=0$ for $x$ not in $Z$ and, therefore, that $\lim _{t \rightarrow 0} u(x, t)=0$ for $x$ not in $E$.

To show that $u(x, t)$ meets conditions (i) and (iii), we set $\phi(y)=\int_{-\infty}^{\infty} e^{i x y} d \mu(x)$ and observe that

$$
\Phi(R)=\int_{-R}^{R}|\phi(y)| d y=o(R) \quad \text { as } R \rightarrow \infty
$$

$[8$, p. 261]. It follows from (1) that

$$
u(x, t)=(2 \pi)^{-1} \int_{-\infty}^{\infty} \phi(y) \exp \left[-y^{2} t\right] e^{-i y x} d y \quad \text { for } t>0
$$

and consequently that

$$
\|u(x, t)\|_{\infty} \leqq(2 \pi)^{-1} \int_{0}^{\infty} \Phi(y) 2 y t \exp \left[-y^{2} t\right] d y=o\left(t^{-1 / 2}\right) \quad \text { as } t \rightarrow 0 .
$$

We conclude that $u(x, t)$ meets both conditions (i) and (iii) and, since

$$
\int_{-\infty}^{\infty} u(x, t) d x=1 \quad \text { for } t>0
$$

that condition (ii) as stated is in a certain sense best possible.

To show that condition (iii) of Theorem 3 cannot be weakened to read "for every $x$ in $E$ but one," we observe that $\|k(x, t)\|_{\infty}=o\left(t^{-1}\right)$ as $t \rightarrow 0, \lim _{t \rightarrow 0} k(x, t)=0$ for $x \neq 0, \lim _{t \rightarrow 0} t^{1 / 2} k(o, t)=(4 \pi)^{-1 / 2} \neq 0$, and, therefore, that condition (iii) as stated is in a certain sense best possible.

We shall prove Theorem 1 in this paper, but before proceeding to the proof let us observe first that Theorem 1 contains the classical Tychonoff theorem on the uniqueness of solutions of the heat equation in an infinite strip, [6, p. 88] or [5, p. 206]. However, Theorem 1 neither contains nor is contained in the uniqueness theorems due to Täcklind [4] and Widder [6]. For further comments concerning the uniqueness of solutions of the heat equation in an infinite strip, see the introduction of the last mentioned paper. 
Theorem 1 above was motivated by the author's previous result concerning the uniqueness of functions harmonic in the interior of the unit disc [3]. It was also motivated by the classical theory of trigonometric series, in particular Chapter 9 of [7]. A number of lemmas to be proved in the sequel will have their trigonometric analogues either in [3], [7], or in [8]. However, in the sequel, the theory of trigonometric series will not be used in any explicit manner.

2. A sketch of the proof of a special case of Theorem 3. Before proceeding to the statements and proofs of the various lemmas involved in the proof of Theorem 1, we shall give a sketch of the proof of a special case of Theorem 3 based on these lemmas. This sketch will enable the reader to understand a number of the technical difficulties that will arise in the proof of Theorem 1.

We shall sketch the proof of Theorem 3 in the special case when $E$ is the empty set in (ii), i.e., when (ii) is replaced by (ii') where

(ii') $\lim _{t \rightarrow 0} u(x, t)=0$ for every $x$.

To show that $u(x, t)=0$ in the strip $0<t<c$, it is sufficient to show that $u(x, t)=0$ in the strip $0<t<t_{1}$ where $0<t_{1}<c$. In order to do this, we set for $0<t<t_{1}$,

$$
F(x, t)=-\int_{t}^{t_{1}} u(x, s) d s+\int_{0}^{x} \int_{0}^{y} u\left(z, t_{1}\right) d z d y
$$

and observe that $F(x, t)$ is a solution of the heat equation in the strip $0<t<t_{1}$. We furthermore observe that there is a constant $K_{1}$ such that

$$
|F(x, t)| \leqq K_{1}\left(x^{2}+1\right) t^{-1 / 2}
$$

for $0<t<t_{1}$ and $-\infty<x<\infty$. Also, it follows from (ii') that $\lim _{t \rightarrow 0} F(x, t)=F(x)$ exists and is finite for every $x$. If we can show that $F(x)$ is a linear function, it will follow from Lemma 14 that $F(x, t)$ is independent of $t$ for $0<t<t_{1}$, and consequently that

$$
0=F_{t}(x, t)=u(x, t) \quad \text { for } 0<t<t_{1} .
$$

So this special case of Theorem 3 will be established once it is shown that $F(x)$ is a linear function.

To show that $F(x)$ is a linear function it is sufficient to show that $F(x)$ is a continuous function, for it follows from (32) of Lemma 10 that if $F(x)$ is continuous for $a<x<b$ then

$$
D_{*}^{2} F(x) \leqq 0 \leqq D^{* 2} F(x) \quad \text { for } a<x<b,
$$

and consequently by Lemma 5 that $F(x)$ is a linear function for $a<x<b$.

We let $Z$ designate the set of discontinuities of $F(x)$. It follows from the Baire category theory (in particular from [7, (i) of Theorem 12.3, p. 29] or from [3, Lemma 4, p. 645]), and from (ii') (see the proof of Theorem 1 for the details) that $Z$ is a nowhere dense set on the real line. 
Next, we observe from the proof of Lemma 11 that $u_{x}(x, t)$ is bounded in any substrip of the form $0<t_{0} \leqq t<c$ and furthermore that $\left\|u_{x}(x, t)\right\|_{\infty}=o\left(t^{-3 / 2}\right)$ as $t \rightarrow 0$. Consequently, on choosing a $t_{2}$ such that $0<t_{2}<t_{1}$ and setting

$$
G(x, t)=-\int_{t}^{t_{2}} F_{x}(x, s) d s+\int_{0}^{x} F\left(y, t_{2}\right) d y \quad \text { for } 0<t<t_{2}
$$

we have by Lemma 12 that $\lim _{t \rightarrow 0} G(x, t)=G(x)$ exists and is continuous on $(-\infty, \infty)$. We furthermore have from Lemma 12 that $G(x)$ is smooth on $(-\infty, \infty)$. (Smoothness is the crucial notion in this paper and is defined on [7, p. 43]. See also [7, p. 375].) Also, it follows from Lemma 12 that if $F(x)$ is continuous in $a<x<b$ then $d G(x) / d x$ exists and equals $F(x)$.

Now we already know that if $F(x)$ is continuous in a finite interval $a<x<b$, then $F(x)$ is linear in this interval. Consequently, it follows from the remarks of the preceding paragraph and the last statement in Lemma 12 that

if $F(x)$ is continuous in the finite open interval $(a, b)$,

it is continuous in the closed interval $[a, b]$.

But then it follows immediately that the nowhere dense set $Z$ can have no isolated points and consequently that $\bar{Z}$ is a perfect nowhere dense set.

We use the Baire category theory once again, i.e., [7, (i) of Theorem 12.3, p. 29] or [3, Lemma 4, p. 645], and obtain that if $\bar{Z}$ is nonempty, then there exists an open interval $J$ such that $J \bar{Z} \neq 0$ and such that $F$ is a continuous function when restricted to the set $J \bar{Z}$. But we already know that $F$ is linear in each open interval contiguous to $\bar{Z}$ and is continuous in the closure of each open interval contiguous to $\bar{Z}$. We consequently conclude after an elementary argument that $F$ is a continuous function in the open interval $J$. But then $J \bar{Z}$ is the empty set, and we have arrived at a contradiction. Consequently, $\bar{Z}$ must be empty.

We conclude that $F(x)$ is continuous on the real line, and therefore $F(x)$ is a linear function on the real line. As we have shown earlier, this fact completes the sketch to the proof of the theorem under consideration.

3. Fundamental lemmas. We take as Lemma 1 the following lemma which is essentially a corollary to Tychonoff's uniqueness theorem [6, p. 88] or [5, p. 206].

LEMMA 1. Let $u(x, t)$ be a solution of the heat equation in the strip $0<t<c$. Suppose that

(i) $|u(x, t)| \leqq K \exp \left[\alpha x^{2}\right]$ for $0<t<c$

where $K$ and $\alpha$ are positive constants;

(ii) $\lim _{t \rightarrow 0} u(x, t)=0$ uniformly on compact subsets of $(-\infty, \infty)$.

Then $u(x, t)$ is identically zero in the strip $0<t<c$. 
If $F(x)$ is a function in $L^{1}$ in a neighborhood of the point $x_{0}$ (though not necessarily finite-valued in this neighborhood), we shall set

$$
D^{* 2} F\left(x_{0}\right)=\limsup _{h \rightarrow 0}\left[(2 h)^{-1} \int_{-h}^{h} F\left(x_{0}+x\right) d x-F\left(x_{0}\right)\right] / h^{2} 6^{-1}
$$

with $D_{*}^{2} F\left(x_{0}\right)$ designating the corresponding lim inf. It is clear that if $F(x)$ is in class $C^{2}$ in a neighborhood of $x_{0}$ and $F^{\prime \prime}(x)=d^{2} F(x) / d x^{2}$ then

$$
D_{*}^{2} F\left(x_{0}\right)=D^{* 2} F\left(x_{0}\right)=F^{\prime \prime}\left(x_{0}\right) .
$$

The first lemma that we prove is the following:

LEMMA 2. Let $F(x)$ be a finite-valued measurable function defined on $(-\infty, \infty)$ satisfying the condition $|F(x)| \leqq K \exp \left[\alpha x^{2}\right]$ where $K$ and $\alpha$ are positive constants. For $0<t<(4 \alpha)^{-1}$, set $F(x, t)=\int_{-\infty}^{\infty} k(x-y, t) F(y) d y$. Define

$$
\Delta^{*} F(x)=\limsup _{t \rightarrow 0}[F(x, t)-F(x)] / t
$$

and let $\Delta_{*} F(x)$ designate the corresponding lim inf. Then at each point $x_{0}$, for which $\lim _{t \rightarrow 0} F\left(x_{0}, t\right)=F\left(x_{0}\right)$, the following inequalities hold

$$
D_{*}^{2} F\left(x_{0}\right) \leqq \Delta_{*} F\left(x_{0}\right) \leqq \Delta^{*} F\left(x_{0}\right) \leqq D^{* 2} F\left(x_{0}\right)
$$

It is clear that all of the inequalities in (2) will be established once the first inequality in (2) is established. Furthermore, it is clear that to establish the first inequality in (2), we need only establish it in the special case $x_{0}=0$, i.e., (2) will be established once we show that $\lim _{t \rightarrow 0} F(0, t)=F(0)$ implies that

$$
D_{*}^{2} F(0) \leqq \Delta_{*} F(0)
$$

If $D_{*}^{2} F(0)=-\infty$, (3) is established. We can therefore suppose that $D_{*}^{2} F(0)>q$. (3) will be established if we can show that

$$
\Delta_{*} F(0) \geqq q .
$$

We now establish (4). First choose $\delta$ such that for $0<h \leqq \delta$,

$$
\int_{-h}^{h}[F(x)-F(0)] d x>q 3^{-1} h^{3}
$$

Then for $0<t<(4 \alpha)^{-1}$,

(5) $[F(0, t)-F(0)] / t=\left(4 \pi t^{3}\right)^{-1 / 2} \int_{0}^{\infty}[F(x)+F(-x)-2 F(0)] \exp \left[-x^{2} / 4 t\right] d x$.

Since

$$
t^{-3 / 2} \int_{\delta}^{\infty} \exp \left[(\alpha-1 / 4 t) x^{2}\right] d x \rightarrow 0 \quad \text { as } t \rightarrow 0
$$


we conclude from (5) after integrating by parts that

$$
\begin{aligned}
\Delta_{*} F(0) & \geqq q \liminf _{t \rightarrow 0} \int_{0}^{\delta} 3^{-1} x^{3} x(2 t)^{-1} \exp \left[-x^{2} / 4 t\right] d x /\left(4 \pi t^{3}\right)^{1 / 2} \\
& \geqq 8 q \int_{0}^{\infty} x^{4} \exp \left[-x^{2}\right] d x / 3 \pi^{1 / 2} \\
& \geqq q
\end{aligned}
$$

which fact establishes (4) and consequently the lemma.

Lemma 3. Let $F(x)$ be a function in $L^{1}$ in $(-\infty, \infty)$. For $t>0$, set

$$
F(x, t)=\int_{-\infty}^{\infty} k(x-y, t) F(y) d y
$$

Then at each point $x_{0}$ for which $\lim _{t \rightarrow 0} F\left(x_{0}, t\right)=F\left(x_{0}\right)$ (where $F\left(x_{0}\right)$ is finite or infinite), the following inequalities hold:

$$
D_{*}^{2} F\left(x_{0}\right) \leqq \Delta_{*} F\left(x_{0}\right) \leqq \Delta^{*} F\left(x_{0}\right) \leqq D^{* 2} F\left(x_{0}\right),
$$

where $\Delta_{*} F\left(x_{0}\right)$ and $\Delta^{*} F\left(x_{0}\right)$ are defined as in Lemma 2 .

If $F\left(x_{0}\right)$ is infinite, the proof of this lemma is immediate. If $F\left(x_{0}\right)$ is finite, the proof of this lemma is the same as the proof of Lemma 2 except that we use the additional fact

$$
\begin{aligned}
t^{-3 / 2} \int_{\delta}^{\infty}|F(x)+F(-x)| \exp \left[-x^{2} / 4 t\right] d x & \leqq t^{-3 / 2} e^{-\delta^{2} / 4 t} \int_{-\infty}^{\infty}|F(x)| d x \\
& =o(1) \quad \text { as } t \rightarrow 0 .
\end{aligned}
$$

LEMMA 4. Let $F(x)$ be a finite-valued function defined on the interval $(-\infty, \infty)$ satisfying the condition $|F(x)| \leqq K \exp \left[\alpha x^{2}\right]$ where $K$ and $\alpha$ are positive constants. For $0<t<(4 \alpha)^{-1}$, set $F(x, t)=\int_{-\infty}^{\infty} k(x-y ; t) F(y) d y$. Suppose $F(x)$ is a polynomial of degree $2 n-1, n \geqq 1$, in the open interval $(a, b)$. Then $\lim _{t \rightarrow 0} \partial^{n} F(x, t) / \partial t^{n}=0$ uniformly in every compact subinterval of $(a, b)$.

Let $a<a^{\prime}<b^{\prime}<b$ and $a^{\prime}-a>\delta$ and $b-b^{\prime}>\delta$ where $\delta>0$. Then for $0<t<(4 \alpha)^{-1}$,

$$
\partial^{n} F(x, t) / \partial t^{n}=\int_{-\infty}^{\infty} F(x-y)\left(\partial^{n} k(y, t) / \partial t^{n}\right) d y
$$

Also, for $a^{\prime} \leqq x \leqq b^{\prime}$ there exists a constant $K^{\prime}$ such that $|F(x-y)| \leqq K^{\prime} \exp \left[2 \alpha y^{2}\right]$. 
Since both integrals

$$
\int_{\delta}^{\infty} \exp \left[2 \alpha y^{2}\right]\left|\partial^{n} k(y, t) / \partial t^{n}\right| d y \text { and } \int_{-\infty}^{-\delta} \exp \left[2 \alpha y^{2}\right]\left|\partial^{n} k(y, t) / \partial t^{n}\right| d y
$$

tend to zero as $t \rightarrow 0$, we conclude from (6) that to show $\partial^{n} F(x, t) / \partial t^{n} \rightarrow 0$ as $t \rightarrow 0$ uniformly for $a^{\prime} \leqq x \leqq b^{\prime}$, we need only show that

(7) $\quad \lim _{t \rightarrow 0} \int_{-\delta}^{\delta} F(x-y)\left(\partial^{n} k(y, t) / \partial t^{n}\right) d y=0, \quad$ uniformly for $a^{\prime} \leqq x \leqq b^{\prime}$.

By hypothesis, $F(x)=\sum_{j=0}^{2 n-1} a_{j} x^{j}$ in the interval $(a, b)$ where each $a_{j}$ is a constant for $j=0, \ldots, 2 n-1$. Consequently, $F(x-y)=\sum_{j=0}^{2 n-1} a_{j}(x-y)^{j}$ for $a^{\prime} \leqq x \leqq b^{\prime}$ and $-\delta \leqq y \leqq \delta$. Furthermore, for $t>0, \partial^{n} k(y, t) / \partial t^{n}=\partial^{2 n} k(y, t) / \partial y^{2 n}$. We conclude (7) will follow, once we show

$$
\lim _{t \rightarrow 0} \int_{-\delta}^{\delta} y^{j} \partial^{2 n} k(y, t) / \partial y^{2 n} d y=0 \quad \text { for } j=0, \ldots, 2 n-1 .
$$

Observing that $y^{i} \partial^{m} k(y, t) /\left.\partial y^{m}\right|_{y=\delta} \rightarrow 0$ as $t \rightarrow 0$ for $i$ and $m$ nonnegative integers, we conclude after integrating by parts $j$ times that the integral in (8) is equal to

$$
o(1)+(-1)^{j} j ! \int_{-\delta}^{\delta} \partial^{2 n-j} k(y, t) / \partial y^{2 n-j} d y
$$

But $0 \leqq j \leqq 2 n-1$. Consequently, the integral in (9) is also $o(1)$ as $t \rightarrow 0$; (8) is established, and the proof of the lemma is complete.

We shall say that the function $F(x)$ is upper semi-continuous in the open interval $(a, b)$ if $-\infty \leqq F(x)<\infty$ for every $x$ in $(a, b)$ and if $\lim _{\sup _{y \rightarrow x}} F(y) \leqq F(x)$ for every $x$ in $(a, b)$. We shall say that $F(x)$ is lower semi-continuous in $(a, b)$ if $-F(x)$ is upper semi-continuous in $(a, b)$.

If $\bar{Z}$ is a perfect set and $\bar{Z}(a, b)$ is nonempty, $F$ is upper semi-continuous on the set $\bar{Z}(a, b)$ will be defined in a similar manner.

We next state a useful lemma concerning upper semi-continuous functions.

LEMMA 5. Suppose that $F(x)$ is upper semi-continuous (but not necessarily finitevalued $)$ in the open interval $(a, b)$ and in $L^{1}$ on every compact subset of $(a, b)$. Suppose also that

(i) $D^{* 2} F(x) \geqq 0$ in $(a, b)-E_{1}$ where $E_{1}$ is a countable set contained in $(a, b)$;

(ii) $\lim \sup _{h \rightarrow 0+}\left[(2 h)^{-1} \int_{-h}^{h} F(x+y) d y-F(x)\right] / h \geqq 0$ for $x$ in $E_{1}$.

Then $F(x)$ is convex in $(a, b)$.

The proof of this lemma is very similar to that given in [7, Lemma 3.20, p. 328], and we therefore omit it.

We next prove the following lemma which is a variant (and in one sense only an improvement) over [7, Lemma 3.23, p. 329]. 
Lemma 6. Let $H(x)$ be continuous and let $F(x)$ be upper semi-continuous in the open interval $(a, b)$. Also, let $E_{1}$ be a countable set contained in $(a, b)$. Suppose that

(i) $F(x)$ is finite-valued in $(a, b)-E_{1}$;

(ii) $F(x)$ is $L^{1}$ on every compact subset of $(a, b)$;

(iii) $D_{*}^{2} H(x) \leqq F(x) \leqq D^{* 2} H(x)$ for $x$ in $(a, b)-E_{1}$; $\lim \sup _{h \rightarrow 0+}\left[(2 h)^{-1} \int_{-h}^{h} H(x+y) d y-H(x)\right] / h \geqq 0$

$$
\geqq \lim \inf _{h \rightarrow 0}\left[(2 h)^{-1} \int_{-h}^{h} H(x+y) d y-H(x)\right] / H \text { for } x \text { in } E_{1} \text {. }
$$

Then if $a<a^{\prime}<b^{\prime}<b$, there exist constants $A$ and $B$ such that

$$
H(x)=\int_{a^{\prime}}^{x} \int_{a^{\prime}}^{y} F(z) d z d y+A x+B \quad \text { for } a^{\prime} \leqq x \leqq b^{\prime} .
$$

To prove the lemma, we set

$$
M(x)=H(x)-\int_{a^{\prime}}^{x} \int_{a^{\prime}}^{y} F(z) d z d y \quad \text { for } a^{\prime} \leqq x \leqq b^{\prime},
$$

and observe that $M(x)$ is continuous in the closed interval $\left[a^{\prime}, b^{\prime}\right]$. Furthermore, the upper semi-continuity of $F$ implies that $D^{* 2} \int_{a^{\prime}}^{x} \int_{a^{\prime}}^{y} F(z) d z d y \leqq F(x)$ for $a^{\prime}<x<b^{\prime}$. Consequently, we obtain from (11), (i) and (iii) that

$$
D^{* 2} M(x) \geqq D^{* 2} H(x)-D^{* 2} \int_{a^{\prime}}^{x} \int_{a^{\prime}}^{y} F(z) d z d y \geqq 0
$$

for $x$ in $\left(a^{\prime}, b^{\prime}\right)-E_{1}\left(a^{\prime}, b^{\prime}\right)$. Also, it follows from (ii) and (iv) that

$$
\limsup _{h \rightarrow 0+}\left[(2 h)^{-1} \int_{-h}^{h} M(x+y) d y-M(x)\right] / h \geqq 0
$$

for $x$ in $E_{1}$. We conclude from this last fact, (12), and Lemma 5 that

$$
\begin{aligned}
& M(x) \text { is convex in the open interval }\left(a^{\prime}, b^{\prime}\right) \text { and } \\
& \text { continuous in the closed interval }\left[a^{\prime}, b^{\prime}\right] .
\end{aligned}
$$

Next, using the upper semi-continuity of $F$ once again, we choose a sequence of functions, $\left\{F_{n}(x)\right\}_{n=1}^{\infty}$, which enjoy the following three properties:

$$
\begin{aligned}
& F_{n}(x) \text { is continuous on }\left[a^{\prime}, b^{\prime}\right] \text { for } n=1,2, \ldots \\
& F_{n}(x) \geqq F_{n+1}(x) \geqq F(x) \text { for } n=1,2, \ldots \\
& \lim _{n \rightarrow \infty} F_{n}(x)=F(x) \text { for } a^{\prime} \leqq x \leqq b^{\prime} .
\end{aligned}
$$

$$
\text { We set } M_{n}(x)=H(x)-\int_{a^{\prime}}^{x} \int_{a^{\prime}}^{y} F_{n}(z) d z d y, \quad n=1,2, \ldots \text {, }
$$

and observe from (14), (15), and (ii) that

$$
D_{*}^{2} M_{n}(x)=D_{*}^{2} H(x)-F_{n}(x) \leqq 0 \quad \text { for } x \text { in }\left(a^{\prime}, b^{\prime}\right)-E_{1}\left(a^{\prime}, b^{\prime}\right) .
$$


Consequently, we conclude from (iv), (18) and Lemma 5 that for each $n$, $-M_{n}(x)$ is convex on the open interval $\left(a^{\prime}, b^{\prime}\right)$ and continuous on the closed interval $\left[a^{\prime}, b^{\prime}\right]$.

But then it follows from (11), (14), (15), (16), and (17) that

$$
\lim _{n \rightarrow \infty}-M_{n}(x)=-M(x) \quad \text { for } a^{\prime} \leqq x \leqq b^{\prime},
$$

and consequently from (19) that

$-M(x)$ is convex on the open interval $\left(a^{\prime}, b^{\prime}\right)$.

The conclusion to the lemma, namely (10), follows from this last fact, (11) and (13).

LEMMA 7. Let $F(x)$ be in $L^{1}$ on $(-\infty, \infty)$, and for $t>0$, set

$$
F(x, t)=\int_{-\infty}^{\infty} k(x-y, t) F(y) d y .
$$

\section{Suppose that}

(i) $F(x)$ is finite-valued in a neighborhood of $x_{0}$;

(ii) $\lim _{t \rightarrow 0} F\left(x_{0}, t\right)=F\left(x_{0}\right)$;

(iii) $\lim _{t \rightarrow 0}\left[F\left(x_{0}+h\right)+F\left(x_{0}-h\right)-2 F\left(x_{0}\right)\right] / h^{2}=\nu$ where $v$ is finite.

Then $\lim _{t \rightarrow 0} \partial F\left(x_{0}, t\right) / \partial t=\nu$.

With no loss in generality, we can take $x_{0}=0, F(0)=0$, and $\nu=0$.

The lemma will be established if we can show given $\varepsilon>0$,

$$
\limsup _{t \rightarrow 0}|\partial F(0, t) / \partial t| \leqq 6 \varepsilon
$$

To establish (20), we choose $\delta>0$ such that

$$
|F(y)+F(-y)| \leqq \varepsilon y^{2} \quad \text { for }|y| \leqq \delta .
$$

Next, we observe that for $t>0$,

$$
F(0, t)=\int_{0}^{\infty}[F(y)+F(-y)] k(y, t) d y,
$$

and consequently since $F$ is in $L^{1}$ on $(-\infty, \infty)$ and $\sup _{\delta \leqq|y|}\left|k_{t}(y, t)\right|=o(1)$ as $t \rightarrow 0$, that

$$
\begin{aligned}
\partial F(0, t) / \partial t & =\int_{0}^{\delta} k_{t}(y, t)[F(y)+F(-y)] d y+o(1) \\
& =\int_{0}^{\delta} k_{y y}(y, t)[F(y)+F(-y)] d y+o(1) .
\end{aligned}
$$

We conclude from (21) that

$$
|\partial F(0, t) / \partial t| \leqq \varepsilon \int_{0}^{\delta} y^{2}\left|k_{y y}(y, t)\right| d y+o(1) .
$$


But then it follows from (22) that (20) will be established once we show that

$$
\limsup _{t \rightarrow 0} \int_{0}^{\delta} y^{2}\left|k_{y y}(y, t)\right| d y \leqq 6 .
$$

We establish (23) by first observing that

$$
k_{y y}(y, t)=\left(4 \pi^{1 / 2} t^{3 / 2}\right)^{-1} \exp \left[-y^{2} / 4 t\right]\left[y^{2} / 2 t-1\right] .
$$

Consequently, we obtain that for $0<(2 t)^{1 / 2}<\delta$,

$$
\int_{0}^{\delta} y^{2}\left|k_{y y}(y, t)\right| d y=-\int_{0}^{(2 t)^{1 / 2}} y^{2} k_{y y}(y, t) d y+\int_{(2 t)^{1 / 2}}^{\delta} y^{2} k_{y y}(y, t) d y .
$$

After integrating by parts several times, we then obtain that the expression on the left in (23) is majorized by

$$
1+\limsup _{t \rightarrow 0}-4 t k_{y}\left[(2 t)^{1 / 2}, t\right]+\limsup _{t \rightarrow 0} 4(2 t)^{1 / 2} k\left[(2 t)^{1 / 2}, t\right]
$$

But this expression is in turn majorized by $1+1+4$; (23) is therefore established, and the proof to the lemma is complete.

LEMMA 8. Let $F(x)$ be in $L^{1}$ on $(-\infty, \infty)$ and for $t>0$, set

$$
F(x, t)=\int_{-\infty}^{\infty} k(x-y, t) F(y) d y .
$$

Suppose that $\lim _{t \rightarrow 0} F\left(x_{0}, t\right)=F\left(x_{0}\right)$ is finite. Then the following inequalities hold:

$$
\begin{gathered}
\liminf _{h \rightarrow 0+}\left[(2 h)^{-1} \int_{-h}^{h} F\left(x_{0}+x\right) d x-F\left(x_{0}\right)\right] / h \\
\leqq 2^{-1} \pi^{1 / 2} \limsup _{t \rightarrow 0} t^{1 / 2} \partial F\left(x_{0}, t\right) / \partial t, \\
\limsup _{h \rightarrow 0+}\left[(2 h)^{-1} \int_{-h}^{h} F\left(x_{0}+x\right) d x-F\left(x_{0}\right)\right] / h \\
\geqq 2^{-1} \pi^{1 / 2} \liminf _{t \rightarrow 0} t^{1 / 2} \partial F\left(x_{0}, t\right) / \partial t .
\end{gathered}
$$

With no loss in generality, we can assume $x_{0}=0$. We also note that once (24) is established, (25) follows immediately. We shall prove (24).

From the extended law of the mean, we obtain that

$$
\frac{F(0, t)-F(0)}{t^{1 / 2}-0}=\left.2 t^{1 / 2} \frac{\partial F(0, t)}{\partial t}\right|_{t=s} \quad \text { where } 0<s<t
$$

and consequently that

$$
\limsup _{t \rightarrow 0}[F(0, t)-F(0)] t^{-1 / 2} \leqq \limsup _{t \rightarrow 0} 2 t^{1 / 2} \partial F(0, t) / \partial t .
$$


If the expression on the left side of the inequality in (24) is $-\infty$, the inequality in (24) holds. Let us suppose therefore that

$$
\liminf _{h \rightarrow 0+}\left[(2 h)^{-1} \int_{-h}^{h} F(x) d x-F(0)\right] / h>q
$$

where $q$ is finite. Then it follows from (26) that (24) holds once we show that

$$
\limsup _{t \rightarrow 0}[F(0, t)-F(0)] t^{-1 / 2} \geqq q 4 \pi^{-1 / 2} .
$$

Using (27) choose $\delta>0$ such that

$$
\int_{0}^{h}[F(x)+F(-x)-2 F(0)] d x>2 q h^{2} \quad \text { for } 0<h<\delta .
$$

Now from this last fact and the fact that $-k_{x}(x, t) \geqq 0$ for $x \geqq 0$, we obtain after integrating by parts that for $t>0$,

$$
\begin{aligned}
{[F(0, t)-F(0)] t^{-1 / 2} } & =t^{-1 / 2} \int_{0}^{\infty}[F(x)+F(-x)-2 F(0)] k(x, t) d x \\
& \geqq 2 q t^{-1 / 2} \int_{0}^{\delta} x^{2}\left[-k_{x}(x, t)\right] d x+o(1)
\end{aligned}
$$

An easy computation shows that

$$
\lim _{t \rightarrow 0} 2 t^{-1 / 2} \int_{0}^{\delta} x^{2}\left[-k_{x}(x, t)\right] d x=4 \pi^{-1 / 2}
$$

But then (28) follows from (29), and the proof to the lemma is complete.

LEMMA 9. Let $F(x)$ be in $L^{1}$ on $(-\infty, \infty)$ and for $t>0$, set

$$
F(x, t)=\int_{-\infty}^{\infty} k(x-y, t) F(y) d y .
$$

Suppose that $\lim _{t \rightarrow 0} F\left(x_{0}, t\right)=F\left(x_{0}\right)$ is finite. Then the following inequalities hold:

$$
\begin{aligned}
D_{*}^{2} F\left(x_{0}\right) & \leqq \limsup _{t \rightarrow 0} \partial F\left(x_{0}, t\right) / \partial t \text { and } \\
D^{* 2} F\left(x_{0}\right) & \geqq \liminf _{t \rightarrow 0} \partial F\left(x_{0}, t\right) / \partial t .
\end{aligned}
$$

For $t>0$, we obtain from the mean-value theorem that

$$
\left[F\left(x_{0}, t\right)-F\left(x_{0}\right)\right] t^{-1}=\partial F\left(x_{0}, t\right) /\left.\partial t\right|_{t=s}, \quad 0<s<t .
$$

Consequently, the following inequalities obtain

$$
\liminf _{t \rightarrow 0} \partial F\left(x_{0}, t\right) / \partial t \leqq \Delta_{*} F\left(x_{0}\right) \leqq \Delta^{*} F\left(x_{0}\right) \leqq \limsup _{t \rightarrow 0} \partial F\left(x_{0}, t\right) / \partial t .
$$

But then (30) follows immediately from these inequalities and Lemma 3. 
We next come to one of the key lemmas of this paper.

LEMMA 10. Let $u(x, t)$ be a solution of the heat equation in the strip $0<t<c$. Let $0<t_{1}<c$ and for $0<t<t_{1}$, set $F(x, t)=-\int_{t}^{t_{1}} u(x, s) d s+\int_{0}^{x} \int_{0}^{y} u\left(z, t_{1}\right) d z d y$. Suppose that

(i) $|u(x, t)| \leqq \varepsilon(t) e^{\alpha x^{2}} t^{-1}$ for $0<t<c$ where $\alpha$ is a positive constant and $\varepsilon(t)$ is bounded for $0<t<c$ and $\varepsilon(t)=o(1)$ as $t \rightarrow 0$;

(ii) $F(x)=\lim _{t \rightarrow 0} F(x, t)$ exists (not necessarily finite) for $a<x<b$;

(iii) $F(x)$ is upper semi-continuous for $a<x<b$;

(iv) $F(x)$ is finite-valued for $x$ in $(a, b)-E_{1}$ where $E_{1}$ is a countable set contained in $(a, b)$.

Then

$$
F(x) \text { is in } L^{1} \text { on every compact subset of }(a, b) \text {; }
$$

$$
\text { if } F\left(x_{0}\right) \text { is finite where } a<x_{0}<b \text {, }
$$

$$
\liminf _{t \rightarrow 0} u\left(x_{0}, t\right) \leqq D^{* 2} F\left(x_{0}\right) \text { and } \limsup _{t \rightarrow 0} u\left(x_{0}, t\right) \geqq D_{*}^{2} F\left(x_{0}\right) \text {; }
$$

if $F\left(x_{0}\right)$ is finite where $a<x_{0}<b$,

$$
\liminf _{h \rightarrow 0+}\left[(2 h)^{-1} \int_{-h}^{h} F\left(x_{0}+x\right) d x-F\left(x_{0}\right)\right] / h \leqq 2^{-1} \pi^{1 / 2} \limsup _{t \rightarrow 0} t^{1 / 2} u\left(x_{0}, t\right)
$$

and

$$
\limsup _{h \rightarrow 0+}\left[2 h^{-1} \int_{-h}^{h} F\left(x_{0}+x\right) d x-F\left(x_{0}\right)\right] / h \geqq 2^{-1} \pi^{1 / 2} \liminf _{t \rightarrow 0} t^{1 / 2} u\left(x_{0}, t\right) ;
$$

if $F(x)$ is finite-valued in a neighborhood of the point $x_{0}$ where

$a<x_{0}<b$ and if $\lim _{h \rightarrow 0}\left[F\left(x_{0}+h\right)+F\left(x_{0}-h\right)-2 F\left(x_{0}\right)\right] / h^{2}=\nu$ where

$\nu$ is finite, $\lim _{t \rightarrow 0} u\left(x_{0}, t\right)=\nu$;

$$
\text { if } F(x) \text { is continuous for } a^{\prime \prime}<x<b^{\prime \prime} \text { where } a<a^{\prime \prime}<b^{\prime \prime}<b \text {, }
$$

$$
\lim _{t \rightarrow 0} F(x, t)=F(x)
$$

uniformly on compact subsets of $\left(a^{\prime \prime}, b^{\prime \prime}\right)$.

We first observe that $F(x, t)$ is a solution of the heat equation in the strip $0<t<c$, and furthermore there exist a positive constant $K^{\prime}$ and a nonnegative bounded function $\varepsilon_{1}(t)$ with $\varepsilon_{1}(t)=o(1)$ as $t \rightarrow 0$ such that

$$
|F(x, t)| \leqq \varepsilon_{1}(t) e^{\alpha x^{2}}\left|\log t^{-1}\right|+K^{\prime} e^{\alpha x^{2}}\left(x^{2}+1\right) \quad \text { for } 0<t<t_{1} .
$$

Next, we choose $t_{2}$ with $0<t_{2}<t_{1}$ and set for $0<t<t_{2}$,

$$
H(x, t)=-\int_{t}^{t_{2}} F(x, s) d s+\int_{0}^{x} \int_{0}^{y} F\left(z, t_{2}\right) d z d y .
$$


We observe, once again, that $H(x, t)$ is a solution of the heat equation for $0<t<t_{2}$ and that by (36) there exists a constant $K^{\prime \prime}$ such that

$$
|H(x, t)| \leqq K^{\prime \prime} \exp \left[2 \alpha x^{2}\right] \quad \text { for } 0<t<t_{2} .
$$

We also obtain from (36) and (37) that

$$
\lim _{t \rightarrow 0} H(x, t)=H(x) \text { uniformly on compact subsets of }(-\infty, \infty)
$$

where

$$
H(x)=-\int_{0}^{t_{2}} F(x, s) d s+\int_{0}^{x} \int_{0}^{y} F\left(z, t_{2}\right) d z d y .
$$

It follows from (38), (39), and Lemma 1 that

$$
H(x, t)=\int_{-\infty}^{\infty} k(x-y, t) H(y) d y \quad \text { for } 0<t<\min \left[t_{2},(8 \alpha)^{-1}\right] .
$$

It follows from (37) and (40) and from (ii) of the lemma that

$$
\Delta^{*} H(x)=\Delta_{*} H(x)=F(x) \quad \text { for } a<x<b .
$$

But then we obtain from (38), (39), (41), (42), and Lemma 2 that

$$
D_{*}^{2} H(x) \leqq F(x) \leqq D^{* 2} H(x) \quad \text { for } a<x<b .
$$

We next show that

$$
\lim _{h \rightarrow 0+}\left[(2 h)^{-1} \int_{-h}^{h} H(x+y) d y-H(x)\right] / h=0 \quad \text { for } x \text { in }(-\infty, \infty) .
$$

We need only establish (44) for a fixed $x_{1}$.

It is clear that (44) will be established for $x_{1}$ if we show

$$
H\left(x_{1}+h\right)+H\left(x_{1}-h\right)-2 H\left(x_{1}\right)=o(h) \quad \text { as } h \rightarrow 0 .
$$

Now the left side of the equality in ( $\left.44^{\prime}\right)$ is equal to

$$
\begin{aligned}
& {\left[H\left(x_{1}+h\right)-H\left(x_{1}+h, h^{2}\right)\right]+\left[H\left(x_{1}-h\right)-H\left(x_{1}-h, h^{2}\right)\right]} \\
& \quad-2\left[H\left(x_{1}\right)-H\left(x_{1}, h^{2}\right)\right]+\left[H\left(x_{1}+h, h^{2}\right)+H\left(x_{1}-h, h^{2}\right)-2 H\left(x_{1}, h^{2}\right)\right] .
\end{aligned}
$$

That the first three terms in $\left(44^{\prime \prime}\right)$ are $o(h)$ as $h \rightarrow 0$ follows immediately from (36), (37), (40) and the fact that

$$
\int_{0}^{h^{2}}|\log t| d t=o(h) \quad \text { as } h \rightarrow 0 .
$$

By (37), the last expression in (44") for $0<h^{2}<t_{2}$ is given by

$$
-\int_{h^{2}}^{t_{2}}\left[F\left(x_{1}+h, s\right)+F\left(x_{1}-h, s\right)-2 F\left(x_{1}, s\right)\right] d s+O\left(h^{2}\right),
$$

since $F\left(x, t_{2}\right)$ is a continuous function of $x$. 
We conclude that $\left(44^{\prime}\right)$ will be established (and consequently (44)) if we show that the integral in $\left(44^{\prime \prime}\right)$ is $o(h)$ as $h \rightarrow 0$.

The fact that $F(x, s)$ is in class $C^{2}$ for $s$ fixed and $0<s<t_{1}$ and the fact that $\partial^{2} F(x, s) / \partial x^{2}=u(x, s)$ implies from (i) of the lemma that

$$
\begin{aligned}
&\left|F\left(x_{1}+h, s\right)+F\left(x_{1}-h, s\right)-2 F\left(x_{1}, s\right)\right| \leqq K^{m} h^{2} s^{-1} \\
& \text { for } 0<s<t_{2} \text { and } 0<h^{2}<t_{2}
\end{aligned}
$$

where $K^{m}$ is a constant.

We conclude from this last fact that the integral in $\left(44^{m}\right)$ is $o(h)$ as $h \rightarrow 0$ and consequently that (44) is established.

To obtain (31) in the conclusion of the lemma, let $a<a_{1}<b_{1}<b$. Then since by (iv) of the lemma $F(x)$ is not identically $-\infty$ in $\left(a_{1}, b_{1}\right)$, it follows from (iii) of the lemma that $F(x)$ takes a finite maximum in $\left[a_{1}, b_{1}\right]$ which we designate by $M$. Therefore by (43), $D_{*}^{2}\left[H(x)-M x^{2} / 2\right]=D_{*}^{2} H(x)-M \leqq 0$ for $x$ in $\left(a_{1}, b_{1}\right)$. Consequently, since $H(x)$ is continuous in $\left(a_{1}, b_{1}\right)$ by (39), we obtain that $M x^{2} / 2-H(x)$ is a convex function in $\left(a_{1}, b_{1}\right)$. But then from [7, p. 328] we obtain that for almost every $x$ in $\left(a_{1}, b_{1}\right)$

$$
\lim _{h \rightarrow 0}[H(x+h)+H(x-h)-2 H(x)] / h^{2} \quad \text { exists and is finite }
$$

and furthermore this limit represents a function which is in $L^{1}$ on every compact subset of $\left(a_{1}, b_{1}\right)$.

We conclude from this fact that $D_{*}^{2} H(x)=D^{* 2} H(x)$ almost everywhere in $\left(a_{1}, b_{1}\right)$ and represents a function which is in $L^{1}$ on every compact subset of $\left(a_{1}, b_{1}\right)$. But then we obtain immediately from (43) that $F(x)$ is in $L^{1}$ on every compact subset of $\left(a_{1}, b_{1}\right)$, which fact establishes (31).

To establish (32), (33), (34), and (35), let

$$
a<a^{\prime}<a^{\prime \prime}<a^{m}<x_{0}<b^{m}<b^{\prime \prime}<b^{\prime}<b
$$

where $F\left(a^{\prime}\right) \neq-\infty$ and $F\left(b^{\prime}\right) \neq-\infty$.

Take $\lambda(x)$ to be a nonnegative continuous function where $\lambda(x)=1$ for $a^{\prime \prime} \leqq x \leqq b^{\prime \prime}$, $0<\lambda(x) \leqq 1$ for $a^{\prime}<x<b^{\prime}$, and $\lambda(x)=0$ for $-\infty<x \leqq a^{\prime}$ and $b^{\prime} \leqq x<\infty$.

Next we set

$$
\begin{aligned}
F_{1}(x) & =\lambda(x) F(x) & & \text { for } a^{\prime}<x<b^{\prime} \\
& =0 & & \text { for }-\infty<x \leqq a^{\prime} \text { and } b^{\prime} \leqq x<\infty
\end{aligned}
$$

It then follows from (iii) of the lemma, (31), (45), and (46) that $F_{1}(x)$ is an upper semi-continuous function which is in $L^{1}$ on $(-\infty, \infty)$ and that $F_{1}(x)$ vanishes outside of a finite interval. 
We set $F_{1}(x, t)=\int_{-\infty}^{\infty} k(x-y, t) F_{1}(y) d y$ for $t>0$ and observe that $F_{1}(x, t)$ is a solution of the heat equation for $t>0$. Also we observe that

$$
\left|F_{1}(x, t)\right| \leqq K_{1} t^{-1 / 2} \quad \text { for } t>0 \text { where } K_{1} \text { is a constant }
$$

and that

$$
\int_{-\infty}^{\infty}\left|F_{1}(x, t)-F_{1}(x)\right| d x \rightarrow 0 \quad \text { as } t \rightarrow 0 .
$$

With $t_{2}$ as in (37), we set for $0<t<t_{2}$

$$
H_{1}(x, t)=-\int_{t}^{t_{2}} F_{1}(x, s) d s+\int_{0}^{x} \int_{0}^{y} F_{1}\left(z, t_{2}\right) d z d y,
$$

and observe from (47) that

$$
\left|H_{1}(x, t)\right| \leqq K_{2}\left(x^{2}+1\right) \quad \text { for } 0<t<t_{2} \text { where } K_{2} \text { is a constant. }
$$

We also observe that $H_{1}(x, t)$ is a solution of the heat equation for $0<t<t_{2}$ and from (47) that

$$
\begin{gathered}
\lim _{t \rightarrow 0} H_{1}(x, t)=H_{1}(x) \quad \text { uniformly on }(-\infty, \infty) \text { where } \\
H_{1}(x)=-\int_{0}^{t_{2}} F_{1}(x, s) d s+\int_{0}^{x} \int_{0}^{y} F_{1}\left(z, t_{2}\right) d z d y .
\end{gathered}
$$

But then it follows from (50), (51), and Lemma 1 that

$$
H_{1}(x, t)=\int_{-\infty}^{\infty} k(x-y, t) H_{1}(y) d y \quad \text { for } 0<t<t_{2} .
$$

Next, we observe from (49) that for $0<t<t_{2}$.

$$
\partial^{2} H_{1}(x, t) / \partial x^{2}=\partial H_{1}(x, t) / \partial t=F_{1}(x, t) .
$$

Consequently, with $a^{\prime \prime}$ as in (45), we have that

$$
H_{1}(x, t)=\int_{a^{\prime \prime}}^{x} \int_{a^{\prime \prime}}^{y} F_{1}(z, t) d z d y+A(t) x+B(t)
$$

where $A(t)$ and $B(t)$ are continuous functions of $t$ in the interval $0<t<t_{2}$.

From (48), we obtain that for each fixed $x$,

$$
\lim _{t \rightarrow 0} \int_{a^{\prime \prime}}^{x} \int_{a^{\prime \prime}}^{y} F_{1}(z, t) d z d y=\int_{a^{\prime \prime}}^{x} \int_{a^{\prime \prime}}^{y} F_{1}(z) d z d y .
$$

But then it follows from (52), (54), and (55) that there are constants $A_{1}$ and $B_{1}$ such that $\lim _{t \rightarrow 0} A(t)=A_{1}$ and $\lim _{t \rightarrow 0} B(t)=B_{1}$. We conclude from (52), (54), and (55) that

$$
H_{1}(x)=\int_{a^{\mu}}^{x} \int_{a^{*}}^{y} F_{1}(z) d z d y+A_{1} x+B_{1}
$$


On the other hand, it follows from (39), (43), (44), (iii) and (iv) of the lemma, (31), and Lemma 6 that

$$
H(x)=\int_{a^{\prime \prime}}^{x} \int_{a^{\prime \prime}}^{y} F(z) d z d y+A x+B \quad \text { for } a^{\prime \prime} \leqq x \leqq b^{\prime \prime}
$$

where $A$ and $B$ are constants.

Next, we set

$$
\begin{aligned}
H_{2}(x, t) & =H(x, t)-H_{1}(x, t) \quad \text { for } 0<t<t_{2}, \\
H_{2}(x) & =H(x)-H_{1}(x)
\end{aligned}
$$

and conclude from (41) and (53) that

$$
H_{2}(x, t)=\int_{-\infty}^{\infty} k(x-y, t) H_{2}(y) d y \quad \text { for } 0<t<\min \left[t_{2},(8 \alpha)^{-1}\right] .
$$

We furthermore conclude from (46), (56), and (57) that

$$
H_{2}(x) \text { is a linear function for } x \text { in }\left[a^{\prime \prime}, b^{\prime \prime}\right] \text {. }
$$

We also conclude from (38), (39), (50), and (51) that there exists a constant $K_{3}$ such that $\left|H_{2}(x)\right| \leqq K_{3} \exp \left[2 \alpha x^{2}\right]$. But then it follows from (45), (59), (60), and Lemma 4 that both

$$
\begin{array}{cc}
\lim _{t \rightarrow 0} \partial H_{2}(x, t) / \partial t=0 & \text { uniformly for } a^{m} \leqq x \leqq b^{m} ; \\
\lim _{t \rightarrow 0} \partial^{2} H_{2}(x, t) / \partial t^{2}=0 & \text { uniformly for } a^{m} \leqq x \leqq b^{m} .
\end{array}
$$

However, from (37), (49), and (58), we obtain that $\partial H_{2}(x, t) / \partial t=F(x, t)-F_{1}(x, t)$ for $0<t<t_{2}$. We consequently conclude from (61) and (62) that

$$
F(x, t)-F_{1}(x, t) \rightarrow 0 \quad \text { as } t \rightarrow 0 \text { uniformly for } a^{m} \leqq x \leqq b^{m} ;
$$

(64) $\partial F(x, t) / \partial t-\partial F_{1}(x, t) / \partial t \rightarrow 0$

as $t \rightarrow 0$ uniformly for $a^{m} \leqq x \leqq b^{m}$.

We recall that

$$
F_{1}(x, t)=\int_{-\infty}^{\infty} k(x-y, t) F_{1}(y) d y \quad \text { for } t>0
$$

where $F_{1}(x)$ is in $L^{1}$ on $(-\infty, \infty)$.

To establish (32) and (33), we have by hypothesis in both situations that

$$
\lim _{t \rightarrow 0} F\left(x_{0}, t\right)=F\left(x_{0}\right) \quad \text { where } F\left(x_{0}\right) \text { is finite. }
$$

But then we obtain from (45), (46), (63), and (66) that

$$
\lim _{t \rightarrow 0} F_{1}\left(x_{0}, t\right)=F_{1}\left(x_{0}\right) \quad \text { where } F_{1}\left(x_{0}\right) \text { is finite. }
$$


However, (65), (67), and Lemma 9 give us that

$$
\begin{aligned}
D_{*}^{2} F_{1}\left(x_{0}\right) & \leqq \limsup _{t \rightarrow 0} \partial F_{1}\left(x_{0}, t\right) / \partial t \quad \text { and } \\
D^{* 2} F_{1}\left(x_{0}\right) & \geqq \liminf _{t \rightarrow 0} \partial F_{1}\left(x_{0}, t\right) / \partial t .
\end{aligned}
$$

Now it follows from (45) and (46) that

$$
D_{*}^{2} F_{1}\left(x_{0}\right)=D_{*}^{2} F\left(x_{0}\right) \text { and } D^{* 2} F_{1}\left(x_{0}\right)=D^{* 2} F\left(x_{0}\right) .
$$

On the other hand, it follows from (45) and (64) that

$$
\begin{gathered}
\liminf _{t \rightarrow 0} \partial F\left(x_{0}, t\right) / \partial t=\liminf _{t \rightarrow 0} \partial F_{1}\left(x_{0}, t\right) / \partial t, \\
\limsup _{t \rightarrow 0} \partial F\left(x_{0}, t\right) / \partial t=\limsup _{t \rightarrow 0} \partial F_{1}\left(x_{0}, t\right) / \partial t .
\end{gathered}
$$

Also, it follows from the definition of $F\left(x_{0}, t\right)$ that

$$
\partial F\left(x_{0}, t\right) / \partial t=u\left(x_{0}, t\right) \quad \text { for } 0<t<t_{1} .
$$

But then (68), (69), and (71) give us that

$$
\begin{aligned}
& D_{*}^{2} F\left(x_{0}\right) \leqq \limsup _{t \rightarrow 0} u\left(x_{0}, t\right) \quad \text { and } \\
& D^{* 2} F\left(x_{0}\right) \geqq \liminf _{t \rightarrow 0} u\left(x_{0}, t\right) .
\end{aligned}
$$

Therefore, (32) is established.

To establish (33), we obtain from (45), (64), and (71) that

$$
\begin{gathered}
\limsup _{t \rightarrow 0} t^{1 / 2} \partial F_{1}\left(x_{0}, t\right) / \partial t=\limsup _{t \rightarrow 0} t^{1 / 2} u\left(x_{0}, t\right), \\
\liminf _{t \rightarrow 0} t^{1 / 2} \partial F_{1}\left(x_{0}, t\right) / \partial t=\liminf _{t \rightarrow 0} t^{1 / 2} u\left(x_{0}, t\right) .
\end{gathered}
$$

On the other hand, it follows from (65), (67), and Lemma 8 that

$$
\begin{gathered}
\liminf _{h \rightarrow 0+}\left[(2 h)^{-1} \int_{-h}^{h} F_{1}\left(x_{0}+x\right) d x-F_{1}\left(x_{0}\right)\right] / h \\
\leqq 2^{-1} \pi^{1 / 2} \limsup _{t \rightarrow 0} t^{1 / 2} \partial F_{1}\left(x_{0}, t\right) / \partial t \\
\limsup _{h \rightarrow 0+}\left[(2 h)^{-1} \int_{-h}^{h} F_{1}\left(x_{0}+x\right) d x-F_{1}\left(x_{0}\right)\right] / h \\
\geqq 2^{-1} \pi^{1 / 2} \liminf _{t \rightarrow 0} t^{1 / 2} \partial F_{1}\left(x_{0}, t\right) / \partial t
\end{gathered}
$$

(33) then follows immediately from (73), (74), and the fact that $F(x)=F_{1}(x)$ in a neighborhood of the point $x_{0}$. 
To establish (34), we have from (ii) of the lemma, (45), (46), (63), and the hypothesis of (34) that

$$
\begin{aligned}
& \lim _{t \rightarrow 0} F_{1}(x, t)=F_{1}(x) \text { in a neighborhood of the point } x_{0}, \\
& F_{1}(x) \text { is finite-valued in a neighborhood of the point } x_{0} \text {, and }
\end{aligned}
$$$$
\lim _{h \rightarrow 0}\left[F_{1}\left(x_{0}+h\right)+F_{1}\left(x_{0}-h\right)-2 F_{1}\left(x_{0}\right)\right] / h^{2}=\nu \text { where } \nu \text { is finite. }
$$

We then obtain from (65), (75), and Lemma 7 that

$$
\lim _{t \rightarrow 0} \partial F_{1}\left(x_{0}, t\right) / \partial t=\nu .
$$

But then it follows immediately from (45), (64), (71), and (76) that

$$
\lim _{t \rightarrow 0} u\left(x_{0}, t\right)=\nu
$$

which fact establishes (34).

To establish (35), it is sufficient by (45) to show

$$
\lim _{t \rightarrow 0} F(x, t)=F(x) \quad \text { uniformly for } a^{m} \leqq x \leqq b^{m} .
$$

Now, it follows from (46) and the hypothesis of $(35)$ that $F_{1}(x)$ is continuous for $a^{\prime \prime}<x<b^{\prime \prime}$. It then follows from (65) and well-known facts that

$$
\lim _{t \rightarrow 0} F_{1}(x, t)=F_{1}(x) \quad \text { uniformly for } a^{m} \leqq x \leqq b^{m} .
$$

But then (77) follows immediately from (63), (78), and that $F_{1}(x)=F(x)$ for $a^{m} \leqq x \leqq b^{m}$. Consequently, (35) is established, and the proof to Lemma 10 is complete.

LEMMA 11. Let $u(x, t)$ be a solution of the heat equation in the strip $0<t<c$. Suppose that

$$
|u(x, t)| \leqq \varepsilon(t) \exp \left[\alpha x^{2}\right] t^{-1} \quad \text { for } 0<t<c
$$

where $\alpha$ is a positive constant and $\varepsilon(t)$ is bounded for $0<t<c$ and $\varepsilon(t)=o(1)$ as $t \rightarrow 0$. Then there exists $t_{1}$ with $0<t_{1}<c$ such that

$$
\left|u_{x}(x, t)\right| \leqq \varepsilon_{1}(t) \exp \left[2 \alpha x^{2}\right] t^{-3 / 2} \quad \text { for } 0<t<t_{1}
$$

where $\varepsilon_{1}(t)$ is bounded for $0<t<t_{1}$ and $\varepsilon_{1}(t)=o(1)$ as $t \rightarrow 0$.

We first observe from Lemma 1 that for $0<t<c$ and $0<s<\min \left[c-t,(4 \alpha)^{-1}\right]$,

$$
u_{x}(x, t+s)=\int_{-\infty}^{\infty} u(y, t) k_{x}(x-y, s) d y
$$


Choose $t_{0}$ such that $0<2 t_{0}<\min \left[c,(8 \alpha)^{-1}\right]$. Then from (79) and the hypothesis of the lemma, we obtain that there exists a constant $K$ such that

$$
\begin{aligned}
& \left|u_{x}(x, t+s)\right| \\
& \quad \leqq K \varepsilon(t) \exp \left[2 \alpha x^{2}\right] t^{-1} \int_{-\infty}^{\infty} \exp \left[2(\alpha-1 / 16 s) y^{2}\right] y \exp \left[-y^{2} / 8 s\right] s^{-3 / 2} d y
\end{aligned}
$$

$$
\text { for } 0<s<t_{0} \text { and } 0<t<t_{0} \text {. }
$$

But then (80) implies

$$
\left|u_{x}(x, t+s)\right| \leqq K_{1} \varepsilon(t) \exp \left[2 \alpha x^{2}\right] t^{-1} s^{-1 / 2} \quad \text { for } 0<s<t_{0} \text { and } 0<t<t_{0},
$$

where $K_{1}$ is a constant.

Setting $s=t$ in (81), we obtain

$$
\left|u_{x}(x, 2 t)\right| \leqq K_{1} \varepsilon(t) \exp \left[2 \alpha x^{2}\right] t^{-3 / 2} \quad \text { for } 0<t<t_{0} .
$$

The conclusion to Lemma 11 follows immediately from (82).

We shall say that $G$ is smooth at the point $x$ if $G(x)$ is defined and finite in a neighborhood of $x$ and if

$$
\lim _{h \rightarrow 0}[G(x+h)+G(x-h)-2 G(x)] / h=0 .
$$

We shall say that a function $G$ is smooth on $(-\infty, \infty)$ if it is smooth at every point $x$. Smoothness is a crucial notion to this paper and will be established for the function $G$ which we shall introduce in Lemma 12. For further theorems concerning smoothness, see [7, pp. 43 and 44].

If $G$ is defined and finite in a neighborhood of the point $x$, we shall set

$$
D^{-} G(x)=\limsup _{h \rightarrow 0}[G(x+h)-G(x-h)] / 2 h .
$$

$D_{-} G(x)$ will designate the corresponding lim inf.

We next prove the following lemma which is basic to this paper.

LEMMA 12. Under the same hypothesis as Lemma 11, let $t_{1}$ be as in the conclusion of Lemma 11. Set $F(x, t)=-\int_{t}^{t_{1}} u(x, s) d s+\int_{0}^{x} \int_{0}^{y} u\left(z, t_{1}\right) d z d y$ for $0<t<t_{1}$. Next, choose $t_{2}$ such that $0<t_{2}<t_{1}$ and set

$$
G(x, t)=-\int_{t}^{t_{2}} F_{x}(x, s) d s+\int_{0}^{x} F\left(y, t_{2}\right) d y \quad \text { for } 0<t<t_{2} .
$$

Then there exists a continuous function $G(x)$ defined on $(-\infty, \infty)$ such that

$$
\lim _{t \rightarrow 0} G(x, t)=G(x) \text { uniformly on compact subsets of }(-\infty, \infty) \text {; }
$$

$$
G(x) \text { is smooth on }(-\infty, \infty) \text {; }
$$

$$
D_{-} G(x) \leqq \liminf _{t \rightarrow 0} F(x, t) \leqq \limsup _{t \rightarrow 0} F(x, t) \leqq D^{-} G(x)
$$

$$
\text { for every } x \text { in }(-\infty, \infty)
$$


To prove the lemma, we first observe that for $0<t<t_{1}$,

$$
F_{x}(x, t)=-\int_{t}^{t_{1}} u_{x}(x, s) d s+\int_{0}^{x} u\left(y, t_{1}\right) d y .
$$

Consequently, it follows from the conclusion of Lemma 11 that

$$
\left|F_{x}(x, t)\right| \leqq \varepsilon_{2}(t) \exp \left[2 \alpha x^{2}\right] t^{-1 / 2}+K_{1} \exp \left[2 \alpha x^{2}\right](|x|+1) \quad \text { for } 0<t<t_{1},
$$

where $K_{1}$ is a positive constant and $\varepsilon_{2}(t)$ is a nonnegative bounded function for $0<t<t_{1}$ and $\varepsilon_{2}(t)=o(1)$ as $t \rightarrow 0$.

We set

$$
G(x)=-\int_{0}^{t_{2}} F_{x}(x, s) d s+\int_{0}^{x} F\left(y, t_{2}\right) d y
$$

and observe from (89) that $G(x)$ is well defined on $(-\infty, \infty)$. Furthermore, it follows from (84) and (89) that $\lim _{t \rightarrow 0} G(x, t)=G(x)$ uniformly on compact subsets of $(-\infty, \infty)$. Consequently, $G(x)$ is a continuous function, and also (85) is established.

To establish (86), fix $x_{0}$ and select a $\delta$ with $0<\delta<t_{2}^{1 / 2}$. Then for $0<h<\delta$,

$$
\begin{aligned}
G\left(x_{0}+h\right)+G\left(x_{0}-h\right)-2 G\left(x_{0}\right)= & G\left(x_{0}+h, h^{2}\right)+G\left(x_{0}-h, h^{2}\right) \\
& -2 G\left(x_{0}, h^{2}\right)+\left[G\left(x_{0}+h\right)-G\left(x_{0}+h, h^{2}\right)\right] \\
& +\left[G\left(x_{0}-h\right)-G\left(x_{0}-h, h^{2}\right)\right]+2\left[G\left(x_{0}, h^{2}\right)-G\left(x_{0}\right)\right] .
\end{aligned}
$$

We conclude from (83) that to show $G$ is smooth at $x_{0}$ and consequently on $(-\infty, \infty)$, we need only establish

$$
\lim _{h \rightarrow 0}\left[G\left(x, h^{2}\right)-G(x)\right] / h=0 \quad \text { uniformly for }\left|x-x_{0}\right| \leqq \delta,
$$

and

$$
G\left(x_{0}+h, h^{2}\right)+G\left(x_{0}-h, h^{2}\right)-2 G\left(x_{0}, h^{2}\right)=o(h) \quad \text { as } h \rightarrow 0 .
$$

To show that (91) holds, we observe from (84) and (90) that

$$
G\left(x, h^{2}\right)-G(x)=\int_{0}^{h^{2}} F_{x}(x, s) d s,
$$

and consequently from (89) that there exists a constant $K_{2}$ such that

$$
\left|G\left(x, h^{2}\right)-G(x)\right| \leqq K_{2}\left[\int_{0}^{h^{2}} \varepsilon_{2}(s) s^{-1 / 2} d s+h^{2}\right] \quad \text { for }\left|x-x_{0}\right| \leqq \delta .
$$

(91) follows immediately from (93).

To establish (92), we observe from (84) that the expression on the left side of the equality in (92) is given by

$$
\begin{aligned}
-\int_{h^{2}}^{t_{2}}\left[F_{x}\left(x_{0}+h, s\right)+F_{x}\left(x_{0}-h, s\right)\right. & \left.-2 F_{x}\left(x_{0}, s\right)\right] d s \\
& +\int_{0}^{h}\left[F\left(x_{0}+y, t_{2}\right)-F\left(x_{0}-y, t_{2}\right)\right] d y .
\end{aligned}
$$


Now, $F\left(x, t_{2}\right)$ is a continuous function of $x$. Consequently, the second integral in (94) is $o(h)$ as $h \rightarrow 0$. Therefore, to establish (92), it remains to show that the first integral in (94) is $o(h)$ as $h \rightarrow 0$. We now do this.

We obtain from (88) and the fact that $u(x, t)$ is a solution of the heat equation that for $0<s<t_{2}$,

$$
\begin{aligned}
F_{x}\left(x_{0}+h, s\right)+F_{x}\left(x_{0}-h, s\right) & -2 F_{x}\left(x_{0}, s\right) \\
= & -\int_{s}^{t_{1}}\left[u_{x}\left(x_{0}+h, r\right)+u_{x}\left(x_{0}-h, r\right)-2 u_{x}\left(x_{0}, r\right)\right] d r \\
& +\int_{0}^{h}\left[u\left(x_{0}+y, t_{1}\right)-u\left(x_{0}-y, t_{1}\right)\right] d y \\
= & -\int_{s}^{t_{1}}\left\{\int_{0}^{h}\left[u_{x x}\left(x_{0}+y, r\right)-u_{x x}\left(x_{0}-y, r\right)\right] d y\right\} d r \\
& +\int_{0}^{h}\left[u\left(x_{0}+y, t_{1}\right)-u\left(x_{0}-y, t_{1}\right)\right] d y \\
= & \int_{0}^{h}\left[u\left(x_{0}+y, s\right)-u\left(x_{0}-y, s\right)\right] d s \\
= & \int_{0}^{h}\left[\int_{x_{0}-y}^{x_{0}+y} u_{x}(x, s) d x\right] d s .
\end{aligned}
$$

We conclude from Lemma 11 and this last fact that

$$
\left|F_{x}\left(x_{0}+h, s\right)+F_{x}\left(x_{0}-h, s\right)-2 F_{x}\left(x_{0}, s\right)\right| \leqq \varepsilon_{3}(s) h^{2} s^{-3 / 2} \text { for } 0<s<t_{2},
$$

where $\varepsilon_{3}(s)$ is bounded for $0<s<t_{2}$ and $\varepsilon_{3}(s)=o(1)$ as $s \rightarrow 0$.

Consequently, it follows from (95) that the absolute value of the first integral in (94) is majorized by

$$
h^{2} o(1) \int_{h^{2}}^{\infty} s^{-3 / 2} d s=o(h) \quad \text { as } h \rightarrow 0 .
$$

(92) is therefore established, and consequently (86) is established.

To establish (87), we first observe from (84) and (89) that there exists a constant $K_{3}$ such that

$$
|G(x, t)| \leqq K_{3} \exp \left[3 \alpha x^{2}\right] \quad \text { for } 0<t<t_{2} .
$$

Since $G(x, t)$ is a solution of the heat equation for $0<t<t_{2}$, we conclude from (96), (85), and Lemma 1 that

$$
G(x, t)=\int_{-\infty}^{\infty} k(x-y, t) G(y) d y \quad \text { for } 0<t<\min \left[t_{2}, 1 / 12 \alpha\right] .
$$

To establish (87), we need only show that the first inequality in (87) holds. We now establish this first inequality by showing that for a fixed $x_{0}$,

$$
D_{-} G\left(x_{0}\right) \leqq \liminf _{t \rightarrow 0} F\left(x_{0}, t\right) \text {. }
$$


If $D_{-} G\left(x_{0}\right)=-\infty,(98)$ is established. Let us assume therefore that

$$
D_{-} G\left(x_{0}\right)>q>-\infty \text {. }
$$

If we can show

$$
\liminf _{t \rightarrow 0} F\left(x_{0}, t\right) \geqq q,
$$

(98) will be established.

To obtain (99), we observe from (84) that for $0<t<t_{2}, G_{x}(x, t)=F(x, t)$ and consequently obtain from (97) that for $0<t<\min \left[t_{2}, 1 / 12 \alpha\right]$

$$
\begin{aligned}
F\left(x_{0}, t\right) & =\int_{-\infty}^{\infty} k_{x}\left(x_{0}-y, t\right) G(y) d y \\
& =\int_{-\infty}^{\infty} G\left(x_{0}-y\right)(\partial k(y, t) / \partial y) d y .
\end{aligned}
$$

Now, since by assumption, $D_{-} G\left(x_{0}\right)>q$, we can choose $\delta$ such that

$$
G\left(x_{0}+h\right)-G\left(x_{0}-h\right)>2 q h \quad \text { for } 0<h<\delta .
$$

From (85) and (96), $|G(y)| \leqq K_{3} \exp \left[3 \alpha y^{2}\right]$. Therefore,

$$
\left\{\int_{-\infty}^{-\delta}+\int_{\delta}^{\infty}\right\} G\left(x_{0}-y\right)(\partial k(y, t) / \partial y) d y=o(1) \quad \text { as } t \rightarrow 0
$$

and we conclude from $(100)$ that for $0<t<\min \left[t_{2}, 1 / 12 \alpha\right]$,

$$
F\left(x_{0}, t\right)=\int_{-\delta}^{\delta} G\left(x_{0}-y\right)(\partial k(y, t) / \partial y) d y+o(1)
$$

But $\partial k(y, t) / \partial y$ is an odd function of $y$ and we obtain from this last fact that for $t$ small,

$$
F\left(x_{0}, t\right)=\int_{0}^{\delta}\left[G\left(x_{0}+y\right)-G\left(x_{0}-y\right)\right][-\partial k(y, t) / \partial y] d y+o(1) .
$$

Observing that for $y>0,-\partial k(y, t) / \partial y>0$, we obtain from (101) that the integral on the right in $\left(101^{\prime}\right)$ majorizes

$$
2 q \int_{0}^{\delta} y[-\partial k(y, t) / \partial y] d y=2 q\left[-\delta k(\delta, t)+\int_{0}^{\delta} k(y, t) d y\right] .
$$

We conclude from this last fact and $\left(101^{\prime}\right)$ that (99) holds. Thus (87) is established and the proof to Lemma 12 is complete.

LEMMA 13. Under the same hypothesis as Lemma 12, suppose that in addition

(i) $\lim \inf _{t \rightarrow 0} t^{1 / 2} u(x, t)=0$ for $a<x<b$;

(ii) $\lim _{t \rightarrow 0} F(x, t)=F(x)$ exists and is finite for $a<x<b$; 
(iii) there exists a function $U(x)$ in class $C^{1}$ on $(-\infty, \infty)$ such that $F(x)-U(x)$ is convex for $a<x<b$.

Then

$$
d F(x) / d x=F^{\prime}(x) \text { exists and is continuous for } a<x<b,
$$

and

$$
D_{-} F^{\prime}(x) \leqq \liminf _{t \rightarrow 0} u(x, t) \leqq \limsup _{t \rightarrow 0} u(x, t) \leqq D^{-} F^{\prime}(x) \quad \text { for } a<x<b \text {. }
$$

With $G(x, t)$ defined by (84), $G(x)$ defined by (90), and the fact that $k(x, t)$ satisfies the heat equation for $t>0$, we obtain from (97) that there is a $t_{3}>0$ such that for $0<t<t_{3}$

$$
G_{t x}(x, t)=\int_{-\infty}^{\infty} G(y) k_{x x x}(x-y, t) d y
$$

Now for $0<t<t_{2}$, we obtain from (84) and the hypothesis of Lemma 12 that $G_{t}(x, t)=F_{x}(x, t)$ and therefore, that $G_{t x}(x, t)=F_{x x}(x, t)=u(x, t)$. We conclude from (104) that

$$
u(x, t)=\int_{-\infty}^{\infty} G(x-y)\left(\partial^{3} k(y, t) / \partial y^{3}\right) d y \quad \text { for } 0<t<t_{3} .
$$

Next with $F(x)$ defined by (ii) of the lemma, we set for $a<x<b$,

$$
D^{\sim} F(x)=\limsup _{h \rightarrow 0+}[F(x+h)-F(x)] / h .
$$

Since $F(x)-U(x)$ is a convex function for $a<x<b$, and letting $U^{\prime}(x)=d U(x) / d x$, it follows from [7, p. 22] that $D^{\sim} F(x)-U^{\prime}(x)$ is a nondecreasing function in the open interval $(a, b)$, that $D^{\sim} F(x)$ is bounded on every compact subset of $(a, b)$, and that $F(x)$ is absolutely continuous on every compact subset of $(a, b)$ with a Radon-Nikodym derivative equal to $D^{\sim} F(x)$. Consequently to establish (102), it is sufficient to show that $D^{\sim} F(x)$ is a continuous function in $(a, b)$.

Let $x_{0}$ be a fixed point with $a<x_{0}<b$. We conclude from the above paragraph and the fact that $U^{\prime}(x)$ is continuous that (102) will be established if we show

$$
\liminf _{y \rightarrow 0+}\left[D^{\sim} F\left(x_{0}+y\right)-D^{\sim} F\left(x_{0}-y\right)\right] \leqq 0 .
$$

Choose $\delta>0$ such that

$$
a<x_{0}-\delta<x_{0}<x_{0}+\delta<b .
$$

Then since $F(x)$ is continuous in the interval $(a, b)$ it follows from (ii) of the present lemma and (35) in the conclusion of Lemma 10 that

$$
\lim _{t \rightarrow 0} F(x, t)=F(x) \quad \text { uniformly for } x_{0}-\delta \leqq x \leqq x_{0}+\delta .
$$


From (84) and the fact that $F(x, t)$ satisfies the heat equation for $0<t<t_{2}$, we have that $G_{x}(x, t)=F(x, t)$ for $0<t<t_{2}$. But then $G(x, t)-G\left(x_{0}-\delta, t\right)=\int_{x_{0}-\delta}^{x} F(y, t) d y$ for $0<t<t_{2}$ and $x_{0}-\delta \leqq x \leqq x_{0}+\delta$. We conclude from (109) and (85) that

$$
G(x)-G\left(x_{0}-\delta\right)=\int_{x_{0}-\delta}^{x} F(y) d y \quad \text { for } x_{0}-\delta \leqq x \leqq x_{0}+\delta .
$$

Consequently, it follows from (105), (110), (85), and (96) that for $0<t<t_{3}$,

$$
\begin{aligned}
u\left(x_{0}, t\right) & =\int_{-\delta}^{\delta} F\left(x_{0}-y\right)\left(\partial^{3} k(y, t) / \partial y^{3}\right) d y+o(1) \\
& =\int_{-\delta}^{\delta} F\left(x_{0}-y\right)\left(\partial^{2} k(y, t) / \partial y^{2}\right) d y+o(1) \\
& =\int_{-\delta}^{\delta} F\left(x_{0}+y\right)\left(\partial^{2} k(y, t) / \partial y^{2}\right) d y+o(1) .
\end{aligned}
$$

Now, as mentioned earlier, $F(x)$ is absolutely continuous on $\left[x_{0}-\delta, x_{0}+\delta\right]$ with Radon-Nikodym derivative $D^{\sim} F(x)$. We conclude from the fact that $\partial k(y, t) / \partial y$ is odd and nonpositive for $y \geqq 0$ and from (111) that for $0<t<t_{3}$

$$
\begin{aligned}
u\left(x_{0}, t\right) & =\int_{-\delta}^{\delta} D^{\sim} F\left(x_{0}+y\right)[-\partial k(y, t) / \partial y] d y+o(1) \\
& =\int_{0}^{\delta}\left[D^{\sim} F\left(x_{0}+y\right)-D^{\sim} F\left(x_{0}-y\right)\right][-\partial k(y, t) / \partial y] d y+o(1) .
\end{aligned}
$$

Suppose (107) does not hold. Then there exists $q>0$ and $\delta_{1}$ with $0<\delta_{1}<\delta$ such that

$$
\left[D^{\sim} F\left(x_{0}+y\right)-D^{\sim} F\left(x_{0}-y\right)\right]>q \text { for } 0<y<\delta_{1} \text {. }
$$

But then we conclude from (112), (113), and the fact that $D^{\sim} F(x)$ is bounded for $-\delta \leqq x-x_{0} \leqq \delta$ that

$$
\begin{aligned}
u\left(x_{0}, t\right) & \geqq q \int_{0}^{\delta_{1}}[-\partial k(y, t) / \partial y] d y+o(1) \\
& \geqq q k(0, t)+o(1) .
\end{aligned}
$$

But then we obtain from (114) that

$$
\liminf _{t \rightarrow 0} t^{1 / 2} u\left(x_{0}, t\right) \geqq q(4 \pi)^{-1 / 2}>0,
$$

which contradicts (i) of the lemma. Consequently, (107) is true and (102) is established.

We now know $D^{\sim} F(x)=F^{\prime}(x)$ for $a<x<b$. We shall establish (103) by showing

$$
\limsup _{t \rightarrow 0} u\left(x_{0}, t\right) \leqq D^{-} F^{\prime}\left(x_{0}\right)
$$

where, as above, $a<x_{0}-\delta<x_{0}+\delta<b$. A similar argument will establish the first inequality in (103). 
From (112), we have for $0<t<t_{3}$

$$
u\left(x_{0}, t\right)=\int_{0}^{\delta}\left[F^{\prime}\left(x_{0}+y\right)-F^{\prime}\left(x_{0}-y\right)\right][-\partial k(y, t) / \partial y] d y+o(1) .
$$

If $D^{-} F^{\prime}\left(x_{0}\right)=+\infty,(115)$ is established. Suppose then that $D^{-} F^{\prime}\left(x_{0}\right)<M<+\infty$. (115) will be established if we can show

$$
\limsup _{t \rightarrow 0} u\left(x_{0}, t\right) \leqq M
$$

We choose $\delta_{1}>0$ and $0<\delta_{1}<\delta$ such that

$$
F^{\prime}\left(x_{0}+y\right)-F^{\prime}\left(x_{0}-y\right)<2 M y \text { for } 0<y<\delta_{1} \text {. }
$$

Then it follows from the fact that $F^{\prime}(x)$ is bounded in $\left[x_{0}-\delta, x_{0}+\delta\right]$, from (116), and from (118) that for $0<t<t_{3}$

$$
\begin{aligned}
u\left(x_{0}, t\right) & \leqq 2 M \int_{0}^{\delta_{1}} y[-\partial k(y, t) / \partial y] d y+o(1) \\
& \leqq 2 M \int_{0}^{\delta_{1}} k(y, t) d y+o(1)
\end{aligned}
$$

But then

$$
\limsup _{t \rightarrow 0} u\left(x_{0}, t\right) \leqq 2 M \limsup _{t \rightarrow 0} \int_{0}^{\infty} k(y, t) d y=M
$$

(117) is established; and the proof to (103) and, consequently, the lemma is complete.

LEMMA 14. Let $u(x, t)$ be a solution of the heat equation in the strip $0<t<c$. Suppose that

(i) there exist positive constants $\delta, \alpha$ and $K$ such that $|u(x, t)| \leqq K \exp \left[\alpha x^{2}\right] t^{-1+\delta}$ for $0<t<c$;

(ii) $\lim _{t \rightarrow 0} u(x, t)=A x+B$ where $A$ and $B$ are constants.

Then $u(x, t)=A x+B$ for $0<t<c$.

To prove the lemma, it is enough to show by Lemma 1 that there exists $t_{1}$ with $0<t_{1}<c$ such that

$$
u(x, t)=A x+B \quad \text { for } 0<t<t_{1} .
$$

Choose a $t_{1}$ with $0<t_{1}<c$ and set

$$
F(x, t)=-\int_{t}^{t_{1}} u(x, s) d s+\int_{0}^{x} \int_{0}^{y} u\left(z, t_{1}\right) d z d y .
$$


Then $F(x, t)$ is a solution of the heat equation in the strip $0<t<t_{1}$. Furthermore, since $\delta>0$, it follows from (i) of the lemma and (120) that there exists a function $F(x)$ which is continuous for $-\infty<x<\infty$ such that

$$
\lim _{t \rightarrow 0} F(x, t)=F(x) \quad \text { uniformly on compact subsets of }(-\infty, \infty) .
$$

But then it follows from (i) and (ii) of the lemma, (120), (121), and (32) of Lemma 10 that

$$
D_{*}^{2} F(x) \leqq A x+B \leqq D^{* 2} F(x) \quad \text { for }-\infty<x<\infty .
$$

This last fact and Lemma 6 imply that there exist constants $A_{1}$ and $B_{1}$ such that

$$
F(x)=A x^{3} / 6+B x^{2} / 2+A_{1} x+B_{1} \quad \text { for }-\infty<x<\infty .
$$

Next for $0<t<t_{1}$, we set

$$
V(x, t)=F(x, t)-F(x)-A x t-B t
$$

and obtain from $(122)$ that $V(x, t)$ is a solution of the heat equation in the strip $0<t<t_{1}$. Also, it follows from (i) of the lemma and (120) that there exists a constant $K_{1}$ such that $|F(x, t)| \leqq K_{1} \exp \left[2 \alpha x^{2}\right]$ for $0<t<t_{1}$. But then from (122) and (123) we obtain that there exists a constant $K_{2}$ such that $|V(x, t)| \leqq K_{2} \exp \left[2 \alpha x^{2}\right]$ for $0<t<t_{1}$. We conclude from (121), (123), and Lemma 1 that $V(x, t)=0$ for $0<t<t_{1}$ and therefore, from (123) that

$$
F(x, t)=F(x)+A x t+B t \quad \text { for } 0<t<t_{1} .
$$

On the other hand, from (120) we obtain that $\partial F(x, t) / \partial t=u(x, t)$ for $0<t<t_{1}$ and consequently from (124) that

$$
u(x, t)=A x+B \quad \text { for } 0<t<t_{1} .
$$

(119) is therefore established, and the proof to Lemma 14 is complete.

LEMMA 15. Let $u(x, t)$ be a solution of the heat equation in the strip $0<t<c$. Suppose that

(i) there exist positive constants $\alpha$ and $K$ such that $|u(x, t)| \leqq K_{1} \exp \left[\alpha x^{2}\right] t^{-1 / 2}$ for $0<t<c$;

(ii) $\lim _{t \rightarrow 0} u(x, t)=u(x)$ where $u(x)$ is continuous on $(-\infty, \infty)$;

(iii) there exist positive constants $K_{1}$ and $\beta$ such that $|u(x)| \leqq K \exp \left[\beta x^{2}\right]$.

Then

$$
u(x, t)=\int_{-\infty}^{\infty} k(x-y, t) u(y) d y \quad \text { for } 0<t<\min \left[c,(4 \beta)^{-1}\right]
$$

To prove the lemma, we set

$$
u_{0}(x, t)=\int_{-\infty}^{\infty} k(x-y, t) u(y) d y \quad \text { for } 0<t<(4 \beta)^{-1},
$$


and take $t_{0}$ such that $0<t_{0}<\min \left[c,(4 \beta)^{-1}\right]$. The proof to the lemma will be complete if we can show

$$
u(x, t)=u_{0}(x, t) \quad \text { for } 0<t<t_{0} .
$$

As is well known, the continuity of $u(x)$ and (iii) of the lemma imply that

$$
\lim _{t \rightarrow 0} u_{0}(x, t)=u(x), \quad-\infty<x<\infty .
$$

Also, (125), (iii) of the lemma, and the fact that $t_{0}<(4 \beta)^{-1}$ imply that there exists positive constants $K_{2}$ and $\gamma$ such that

$$
\left|u_{0}(x, t)\right| \leqq K_{2} \exp \left[\gamma x^{2}\right] t^{-1 / 2} \quad \text { for } 0<t<t_{0} .
$$

But then on setting

$$
u_{1}(x, t)=u(x, t)-u_{0}(x, t) \quad \text { for } 0<t<t_{0},
$$

we see from (i) of the lemma and (128) and from (ii) of the lemma and (127) that $u_{1}(x, t)$ meets the conditions in the hypothesis of Lemma 14 with $\delta=1 / 2$ and $A=B=0$. We conclude, consequently, from Lemma 14 , that

$$
u_{1}(x, t)=0 \quad \text { for } 0<t<t_{0} .
$$

(129) and (130) give (126), and the proof to Lemma 15 is complete.

4. Proof of Theorem 1. To prove Theorem 1, we choose $t_{1}$ as in the conclusion of Lemma 11 and also such that $0<t_{1}<\min \left[c,(4 \beta)^{-1}\right]$. Next, for $0<t<t_{1}$, we set

$$
F(x, t)=-\int_{t}^{t_{1}} u(x, s) d s+\int_{0}^{x} \int_{0}^{y} u\left(z, t_{1}\right) d z d y
$$

and observe that $F(x, t)$ is a solution of the heat equation for $0<t<t_{1}$. Furthermore, from (i) of Theorem 1 , we see that there exists a constant $K_{1}$ such that

$$
|F(x, t)| \leqq K_{1} \exp \left[2 \alpha x^{2}\right] t^{-1 / 2} \quad \text { for } 0<t<t_{1} .
$$

Now, it follows from (ii) and (v) of Theorem 1 and (131) that $F(x)$ exists for every $x$ where

$$
F(x)=\lim _{t \rightarrow 0} F(x, t)
$$

and that $F(x)$ is either finite or $-\infty$.

If we can show, with $U(x)$ given by (iv) of Theorem 1 , that

$$
F(x)=U(x)+A x+B \text { for }-\infty<x<\infty
$$

where $A$ and $B$ are constants, then the conclusion of Theorem 1 follows easily.

For using (iv) of Theorem 1, (131), (132), (133), and (134), we see that all the conditions in the hypothesis of Lemma 15 are met and consequently that

$$
F(x, t)=\int_{-\infty}^{\infty} k(x-y, t)[U(y)+A y+B] d y, \quad 0<t<t_{1} .
$$


Since $t_{1}<(4 \beta)^{-1}$, it follows from (iv) of Theorem 1 and (131) on applying $\partial / \partial t$ to both sides of (135) that

$$
u(x, t)=\int_{-\infty}^{\infty} k_{t}(x-y, t) U(y) d y \quad \text { for } 0<t<t_{1} .
$$

But using (i) and (iv) of Theorem 1 and (136), we obtain from Lemma 1 that

$$
u(x, t)=\int_{-\infty}^{\infty} k_{t}(x-y, t) U(y) d y \quad \text { for } 0<t<\min \left[c,(4 \beta)^{-1}\right]
$$

which establishes Theorem 1.

Consequently, to prove Theorem 1 we have to establish (134). To do this, we first prove the following fact:

if $F(x)$ is upper semi-continuous in the open interval $(a, b)$, then $F(x)-U(x)$ is linear in $(a, b)$.

We recall that our definition of upper semi-continuous does not subsume the finiteness of $F$, but only that $-\infty \leqq F(x)<\infty$ for $a<x<b$. However, we do know from (ii) of Theorem 1 and from the definition of $F(x)$, namely (131) and (133), that

$$
F(x) \text { is finite-valued in }(a, b)-E(a, b) .
$$

To establish (138), we use the Vitali-Carathéodory theorem [2, p. 75] for the function $u_{*}(x)=\lim _{\inf _{t \rightarrow 0}} u(x, t)$ and obtain also using (iii) of Theorem 1 a nondecreasing sequence of function $\left\{u_{j}(x)\right\}_{j=1}^{\infty}$ with the following properties:

$u_{j}(x)$ is upper semi-continuous on $(-\infty, \infty)$;

$$
\text { each } u_{j}(x) \text { is bounded above on }(-\infty, \infty) \text {; }
$$

$$
u_{j}(x) \leqq u_{j+1}(x) \leqq u_{*}(x) \quad \text { for }-\infty<x<\infty \text {; }
$$

$\lim _{j \rightarrow \infty} u_{j}(x)=u_{*}(x)$ almost everywhere;

if $S$ is a compact subset of $(-\infty, \infty), u_{j}(x)$ is in $L^{1}$ on $S$ and

$$
\lim _{j \rightarrow \infty} \int_{S} u_{j}(x) d x=\int_{S} u_{*}(x) d x .
$$

Setting

$$
U_{f}(x)=\int_{0}^{x} \int_{0}^{y} u_{f}(z) d z d y,
$$

we observe from (143) that $U_{j}(x)$ is continuous on $(-\infty, \infty)$ and furthermore from the upper semi-continuity of $u_{j}$ that

$$
D^{* 2} U_{f}(x) \leqq u_{f}(x) \quad \text { for }-\infty<x<\infty .
$$


Next, we obtain from $\left(138^{\prime}\right)$, from the hypothesis of (138), and from Lemma 10 that

$$
F(x) \text { is in } L^{1} \text { on every compact subset of }(a, b)
$$

and furthermore from the fact that $F(x)$ is either finite or $-\infty$ that

$$
u_{*}(x) \leqq D^{* 2} F(x) \quad \text { for } a<x<b
$$

and furthermore from (ii) and (v) of Theorem 1 that

$$
\limsup _{h \rightarrow 0+}\left[(2 h)^{-1} \int_{-h}^{h} F(x+y) d y-F(x)\right] / h \geqq 0 \quad \text { for } a<x<b .
$$

Consequently, we conclude from (141), (145), (146), and (ii) of Theorem 1 that

$$
D^{* 2}\left[F(x)-U_{j}(x)\right] \geqq u_{*}(x)-u_{j}(x) \geqq 0 \quad \text { for } x \text { in }(a, b)-E(a, b) .
$$

Since

$$
\lim _{h \rightarrow 0}\left[\frac{1}{2 h} \int_{-h}^{h} U_{j}(x+y) d y-U_{j}(x)\right] / h=0,
$$

it follows from (147) that

$$
\limsup _{h \rightarrow 0}\left\{(2 h)^{-1} \int_{-h}^{h}\left[F(x+y)-U_{j}(x+y)\right] d y-\left[F(x)-U_{j}(x)\right]\right\} / h \geqq 0
$$

for $x$ in $E(a, b)$.

We conclude from $\left(145^{\prime}\right),(148)$, and (149), the fact that $F(x)-U_{j}(x)$ is upper semicontinuous in $(a, b)$ and from Lemma 5 that

$$
F(x)-U_{j}(x) \text { is convex in }(a, b) \quad \text { for } j=1,2, \ldots
$$

But from (143), (144), and (iv) of Theorem 1, we obtain that $U_{j}(x) \rightarrow U(x)$ as $j \rightarrow \infty$. Also, (150) tells us that $F(x)$ is finite-valued and continuous in $(a, b)$. Since the limit of a decreasing sequence of convex functions is either convex or identically $-\infty$, we conclude from (150) that

$$
F(x)-U(x) \text { is convex in }(a, b) .
$$

Observing that all the conditions in the hypothesis of Lemma 13 are met, we apply this lemma and obtain that

$$
d F(x) / d x=F^{\prime}(x) \text { is continuous for } a<x<b,
$$

and that

$$
D_{-} F^{\prime}(x) \leqq u_{*}(x) \leqq D^{-} F^{\prime}(x) \quad \text { for } a<x<b
$$


With $U^{\prime}(x)=d U(x) / d x=\int_{0}^{x} u_{*}(y) d y$, it follows from well-known facts concerning convex functions and from (151) and (152) that

$$
F^{\prime}(x)-U^{\prime}(x) \text { is nondecreasing for } a<x<b
$$

and that

$$
\begin{aligned}
& F^{\prime}(x) \text { is of bounded variation on each compact } \\
& \text { subinterval of the open interval }(a, b) \text {. }
\end{aligned}
$$

Next, we use the other half of the Vitali-Carathéodory theorem for the function $u_{*}(x)$ and obtain a nonincreasing sequence of functions $\left\{v_{j}(x)\right\}_{j=1}^{\infty}$ with the following properties:

$$
\begin{aligned}
& v_{f}(x) \text { is lower semi-continuous on }(-\infty, \infty) ; \\
& \text { each } v_{f}(x) \text { is bounded below on }(-\infty, \infty) ; \\
& v_{f}(x) \geqq v_{f+1}(x) \geqq u_{*}(x) \quad \text { for }-\infty<x<\infty ; \\
& \lim _{f \rightarrow \infty} v_{f}(x)=u_{*}(x) \text { almost everywhere; }
\end{aligned}
$$

if $S$ is a compact subset of $(-\infty, \infty), v_{j}(x)$ is in $L^{1}$ on $S$ and

$$
\lim _{j \rightarrow \infty} \int_{S} v_{j}(x) d x=\int_{S} u_{*}(x) d x .
$$

Setting $V_{f}(x)=\int_{0}^{x} \int_{0}^{y} v_{f}(z) d z$, we obtain from (160)

$$
V_{j}^{\prime}(x)=d V_{j}(x) / d x=\int_{0}^{x} v_{j}(y) d y,
$$

and from (156) that

$$
D_{-} V_{j}^{\prime}(x) \geqq v_{j}(x) \quad \text { for }-\infty<x<\infty .
$$

But then it follows from (153), (158), (162), and (ii) of Theorem 1 that

$$
D_{-}\left[F^{\prime}(x)-V_{j}^{\prime}(x)\right] \leqq D_{-} F^{\prime}(x)-D_{-} V_{j}^{\prime}(x) \leqq 0 \quad \text { for } x \text { in }(a, b)-E(a, b) .
$$

Now from (152) and (161), we have that $F^{\prime}(x)-V_{j}^{\prime}(x)$ is continuous for $a<x<b$ and from (155) and (161) that $F^{\prime}(x)-V_{j}^{\prime}(x)$ is of bounded variation on each compact subinterval of the open interval $(a, b)$. We conclude from (163) and from [7, Lemma 8.19, p. 359] that

(164) $F^{\prime}(x)-V_{j}^{\prime}(x)$ is nonincreasing for $a<x<b$ and $j=1,2, \ldots$

Observing from (160) and (161) that

$$
\lim _{y \rightarrow \infty} V_{j}^{\prime}(x)=\int_{0}^{x} u_{*}(y)=U^{\prime}(x),
$$

we obtain from (164) that

$$
F^{\prime}(x)-U^{\prime}(x) \text { is nonincreasing for } a<x<b .
$$


We conclude from (154) and (165) that

$$
F^{\prime}(x)-U^{\prime}(x) \text { is constant for } a<x<b,
$$

and consequently that

$$
F(x)-U(x) \text { is linear for } a<x<b .
$$

We have therefore established (138).

We next establish the following fact:

if $F(x)$ is continuous in the finite open interval $(a, b)$,

$$
\text { then } F(x) \text { is continuous in the closed interval }[a, b] \text {. }
$$

Using (138) and the fact that $U(x)$ is continuous on $(-\infty, \infty)$, it follows that both

$$
\begin{gathered}
F(a+) \text { and } F(b-) \text { exist and are finite where } \\
\lim _{x \rightarrow a+} F(x)=F(a+) \text { and } \lim _{x \rightarrow b-} F(x)=F(b-) .
\end{gathered}
$$

We shall establish (166) by showing that

$$
F(a)=F(a+)
$$

A similar proof will prevail to show that $F(b)=F(b-)$.

With $t_{1}$ as in (131), i.e., $0<t_{1}<\min \left[c,(4 \beta)^{-1}\right]$ and such that the conclusion of Lemma 11 holds for $t_{1}$, we choose $t_{2}$ such that $0<t_{2}<t_{1}$ and set

$$
G(x, t)=-\int_{t}^{t_{2}} F_{x}(x, s) d x+\int_{0}^{x} F\left(y, t_{2}\right) d y \quad \text { for } 0<t<t_{2} .
$$

Then $G(x, t)$ satisfies the conditions in the hypothesis of Lemma 12; so that in particular we have from (133) and Lemma 12 that

there exists a function $G(x)$ such that

(i) $G(x)$ is continuous on $(-\infty, \infty)$;

(ii) $\lim _{t \rightarrow 0} G(x, t)=G(x)$ uniformly on compact subsets of $(-\infty, \infty)$;

(iii) $G(x)$ is smooth on $(-\infty, \infty)$;

(iv) $D_{-} G(x) \leqq F(x) \leqq D^{-} G(x)$ for $-\infty<x<\infty$.

Now, it follows from the fact that $F(x, t)$ satisfies the heat equation for $0<t<t_{1}$ and from (169) that

$$
G_{x}(x, t)=F(x, t) \quad \text { for } 0<t<t_{2} .
$$

Consequently, for $a<a^{\prime}<b^{\prime}<b$

$$
G\left(b^{\prime}, t\right)-G\left(a^{\prime}, t\right)=\int_{a^{\prime}}^{b^{\prime}} F(x, t) d x, \quad 0<t<t_{2} .
$$


By (ii) of (170), $G\left(b^{\prime}, t\right) \rightarrow G\left(b^{\prime}\right)$ and $G\left(a^{\prime}, t\right) \rightarrow G\left(a^{\prime}\right)$ as $t \rightarrow 0$. By the hypothesis in (166) and by (35) in Lemma $10, F(x, t) \rightarrow F(x)$ uniformly $a^{\prime} \leqq x \leqq b^{\prime}$. We conclude from (171) that

$$
G\left(b^{\prime}\right)-G\left(a^{\prime}\right)=\int_{a^{\prime}}^{b^{\prime}} F(x) d x .
$$

But then using this fact, (167), and (ii) of (170), we conclude on letting $a^{\prime} \rightarrow a$ in (172) that

$$
G\left(b^{\prime}\right)-G(a)=\int_{a}^{b^{\prime}} F(x) d x, \quad a<b^{\prime}<b .
$$

Using (167) once again and (173), we see that

$$
\lim _{h \rightarrow 0+}[G(a+h)-G(a)] / h=F(a+) .
$$

By (iii) of (170), $G(x)$ is smooth at $a$. But then from (174) and the definition of smoothness given in (83), it follows that the derivative of $G(x)$ exists at $a$ and is equal to $F(a+)$. But then,

$$
D_{-} G(a)=D^{-} G(a)=F(a+),
$$

and we conclude from (iv) of (170) that

$$
F(a)=F(a+) \text {. }
$$

(168) is therefore established, and the proof to (166) is complete.

Next, let $R>0$. To establish (134) and consequently Theorem 1 , it is sufficient to show

$$
F(x)-U(x) \text { is linear in the open interval }(-R, R) .
$$

By (138), (175) will be established if we can show

$$
F(x) \text { is upper semi-continuous for }-R<x<R \text {. }
$$

We now establish (176). Let $Z$ be defined by

$$
Z=\{x ;-R<x<R, F \text { is not upper semi-continuous at } x\} .
$$

We propose to show that $Z$ is the empty set. To do this, with $t_{1}$ defined as in (131), we choose a strictly decreasing sequence $\left\{t_{j}^{*}\right\}_{j=1}^{\infty}$ tending to zero, i.e.,

$$
t_{1}=t_{1}^{*}>t_{2}^{*}>\cdots>t_{j}^{*}>\cdots \rightarrow 0,
$$

such that

$$
\left|u(x, t)-u\left(x, t_{j}^{*}\right)\right| \leqq 1 \quad \text { for } t_{j+1}^{*} \leqq t \leqq t_{j}^{*} \text { and }|x| \leqq 2 R .
$$

Next, we observe that if $-R<a<b<R$, there exists a constant $M$ and an open interval $\left(a^{\prime}, b^{\prime}\right)$ with $a<a^{\prime}<b^{\prime}<b$ such that

$$
u\left(x, t_{j}^{*}\right) \geqq M \quad \text { for } a^{\prime}<x<b^{\prime} \text { and } j=1,2, \ldots
$$


This fact follows from (ii) of Theorem 1 and from [3, Lemma 4, p. 645]. But then it follows from (178) and (179) that

$$
u(x, t) \geqq M-1 \quad \text { for } a^{\prime}<x<b^{\prime} \text { and } 0<t<t_{1} .
$$

From (131), we obtain that $F_{t}(x, t)=u(x, t)$ for $0<t<t_{1}$, and consequently from (180) that for each $x$ in $\left(a^{\prime}, b^{\prime}\right)$,

(181) $F(x, t)-(M-1) t$ is a nondecreasing function of $t$ for $0<t<t_{1}$.

But for each $t, F(x, t)-(M-1) t$ is a continuous function of $x$. Furthermore, from (133), $\lim _{t \rightarrow 0} F(x, t)-(M-1) t=F(x)$. We conclude from (181) that

$$
F(x) \text { is upper semi-continuous for } a^{\prime}<x<b^{\prime} \text {, }
$$

and consequently that

$$
Z \text { is nowhere dense in }(-R, R),
$$

where $Z$ is defined in (177).

We next show that

$$
Z \text { has no isolated points. }
$$

Suppose that $z_{0}$ is an isolated point of $Z$. Then there exists an $h>0$ such that $-R<z_{0}-h<z_{0}+h<R$ and such that $F(x)$ is upper semi-continuous in each of the open intervals $\left(z_{0}-h, z_{0}\right)$ and $\left(z_{0}, z_{0}+h\right)$. But then by (138), $F(x)-U(x)$ is linear in each of these open intervals. Since $U(x)$ is continuous on $(-\infty, \infty)$, we conclude that $F(x)$ is continuous in each of the open intervals $\left(z_{0}-h, z_{0}\right)$ and $\left(z_{0}, z_{0}+h\right)$. But then by (166), $F(x)$ is continuous in each of the closed intervals $\left[z_{0}-h, z_{0}\right]$ and $\left[z_{0}, z_{0}+h\right]$. Therefore $F(x)$ is continuous in the closed interval $\left[z_{0}-h, z_{0}+h\right]$. So in particular, $F(x)$ is continuous at $z_{0}$. Therefore, $z_{0}$ is not in $Z$, and we have a contradiction. We conclude that (183) is valid.

Let $\bar{Z}$ designate the closure of $Z$. Then it follows from (182) and (183) that $\bar{Z}$ is either the empty set or a nonempty nowhere dense perfect set contained in the closed interval $[-R, R]$.

We now propose to show that

$$
\bar{Z} \text { is not a nonempty nowhere dense perfect set. }
$$

For suppose that the contrary were true. Then by (ii) of Theorem $1[3$, Lemma 4 , p. 645] and (178) it follows that there is an open interval $(a, b)$ with $-R<a<b<R$ such that

$$
\bar{Z}(a, b) \text { is nonempty }
$$

and a constant $M$ such that

$$
u(z, t) \geqq M \quad \text { for } z \text { in } \bar{Z}(a, b) \text { and } 0<t<t_{1} .
$$


But then from (131) and (186), we obtain that $F_{t}(z, t) \geqq M$ for $z$ in $\bar{Z}(a, b)$ and $0<t<t_{1}$. Consequently for each $z$ in $\bar{Z}(a, b), F(z, t)-M t$ is a nondecreasing function for $0<t<t_{1}$. Also, for each $t$ in the open interval $\left(0, t_{1}\right), F(z, t)-M t$ is a continuous function on the set $\bar{Z}(a, b)$. Furthermore, from (133), we have that $\lim _{t \rightarrow 0} F(z, t)-M t=F(z)$. We conclude that

$F$ is an upper semi-continuous function on the set $\bar{Z}(a, b)$.

We now use (187) to show that $\bar{Z}(a, b)$ is the empty set, thus obtaining a contradiction to (185), and therefore establishing (184).

To show that $\bar{Z}(a, b)$ is the empty set, we need only show that $Z(a, b)$ is the empty set. To show this fact, we need only show that if $z_{0} \in Z(a, b)$, then $F(x)$ is upper semi-continuous at $z_{0}$.

We shall show that $F(x)$ is upper semi-continuous from the right at $z_{0}$. A similar proof will prevail to show that $F(x)$ is upper semi-continuous from the left at $z_{0}$.

Observing once again that $U(x)$ defined in (iv) of Theorem 1 is continuous on $(-\infty, \infty)$, to show that $F(x)$ is upper semi-continuous from the right at $z_{0}$, it is sufficient to show that $F(x)-U(x)$ is upper semi-continuous from the right at $z_{0}$. We establish this fact by showing the following:

Let $N>F\left(z_{0}\right)-U\left(z_{0}\right)$. Then there exists an $h_{0}>0$ such that

$$
F(x)-U(x)<N \quad \text { for } z_{0}<x<z_{0}+h_{0} .
$$

Since we are assuming that $\bar{Z}$ is a nonempty nowhere dense perfect set, one of two situations prevail. Either $z_{0}$ is a left-hand endpoint of an open interval contiguous to $\bar{Z}$ or $z_{0}$ is the limit from the right of a sequence of points from $\bar{Z}$. If the former situation holds then by (138) and (166) there exist $h_{1}>0$ such that $F(x)-U(x)$ is linear in the closed interval $\left[z_{0}, z_{0}+h_{1}\right]$, and (188) follows immediately. Consequently, we need only establish (188) in the latter case, i.e., $z_{0}$ is the limit from the right of a sequence of points from $\bar{Z}$.

Now in this latter case, it follows from (187) and the fact that $F\left(z_{0}\right)-U\left(z_{0}\right)<N$ that there exists $z_{1}$ in $\bar{Z}(a, b)$ such that

$$
F(z)-U(z)<N \quad \text { for } z_{0}<z \leqq z_{1} \text { and } z \text { in } \bar{Z} \text {. }
$$

Set $h_{0}=z_{1}-z_{0}$. Then if $x_{0}$ is in the open interval $\left(z_{0}, z_{0}+h_{0}\right)$ and $x_{0}$ is in $\bar{Z}$, (188) is established. Suppose then that $x_{0}$ is in $\left(z_{0}, z_{0}+h_{0}\right)$ but that $x_{0}$ is not in $\bar{Z}$. Then $x_{0}$ is in some open interval contiguous to $\bar{Z}$ of the form $\left(z_{2}, z_{3}\right)$ with $z_{2}$ and $z_{3}$ in $\bar{Z}$ and $z_{0}<z_{2}<z_{3} \leqq z_{1}$. By (138) and (166), $F(x)-U(x)$ is linear in the closed interval $\left[z_{2}, z_{3}\right]$. Consequently,

$$
F\left(x_{0}\right)-U\left(x_{0}\right) \leqq \max \left[F\left(z_{2}\right)-U\left(z_{2}\right), F\left(z_{3}\right)-U\left(z_{3}\right)\right],
$$

and we conclude from (189) and (190) that $F\left(x_{0}\right)-U\left(x_{0}\right)<N$.

But then (188) is established. Consequently, $F(x)$ is upper semi-continuous in the open interval $(a, b)$, and we have obtained a contradiction to (185). We con- 
clude that (184) holds, and therefore that $Z$ defined in (177) is the empty set. Therefore, (176) holds, and $F(x)-U(x)$ is linear in the open interval $(-R, R)$. But this fact establishes (134), and the proof to Theorem 1 is complete.

\section{BIBLIOGRAPHY}

1. F. Hausdorff, Set theory, Chelsea, New York, 1957.

2. S. Saks, Theory of the integral, Monografie Matematyczne, Vol. 7, Warsaw, 1937.

3. V. L. Shapiro, The uniqueness of functions harmonic in the interior of the unit disc, Proc. London Math. Soc. 13 (1963), 639-652.

4. S. Täcklind, Sur les classes quasianalytiques des solutions des équations aux derivées partielles du type parabolique, Nova Acta Soc. Sci. Uppsalla (4) 10 (1936), 1-57.

5. A. Tychonoff, Théorèmes d'unicité pour l'équation de la chaleur, Mat. Sb. 42 (1935), 199-216.

6. D. V. Widder, Positive temperatures on an infinite rod, Trans. Amer. Math. Soc. 55 (1944), 85-95.

7. A. Zygmund, Trigonometric series, Vol. I, Cambridge Univ. Press, Cambridge, 1959.

8. — , Trigonometric series, Vol. II, Cambridge Univ. Press, Cambridge, 1959.

\section{University of California,}

Riverside, CALIFornia 